\title{
Review Article \\ Metasurfaces-Based Absorption and Reflection Control: Perfect Absorbers and Reflectors
}

\author{
Trevon Badloe, ${ }^{1}$ Jungho Mun, ${ }^{2}$ and Junsuk Rho ${ }^{1,2}$ \\ ${ }^{1}$ Department of Mechanical Engineering, Pohang University of Science and Technology (POSTECH), Pohang 37673, Republic of Korea \\ ${ }^{2}$ Department of Chemical Engineering, Pohang University of Science and Technology (POSTECH), Pohang 37673, Republic of Korea
}

Correspondence should be addressed to Junsuk Rho; jsrho@postech.ac.kr

Received 8 July 2017; Revised 22 September 2017; Accepted 1 October 2017; Published 6 November 2017

Academic Editor: Jingshi Meng

Copyright (C) 2017 Trevon Badloe et al. This is an open access article distributed under the Creative Commons Attribution License, which permits unrestricted use, distribution, and reproduction in any medium, provided the original work is properly cited.

In the past decade, the realisation of negative index materials has initiated extensive research into metamaterials. Perfect absorbers and reflectors are of particular interest as their usefulness is endless in a range of different fields and devices. Since it was originally shown that a device can achieve unity absorption of electromagnetic waves, it has become a hot area of research to develop perfect absorbers based on polarisation independence and incident angle independence, at a range of frequencies from microwave to optical ones. The amazing performance, flexibility, and tunability of these metamaterials will be discussed here, by presenting the different designs and working mechanisms that have been realised up to now. Their limitations and shortcomings will be addressed and future plans for perfect absorbers and reflectors will be suggested.

\section{Introduction}

The control of lights amplitude, phase, and polarisation states through optical devices is a key tool in the modern world for many real-life applications such as sensing [1], imaging [2], and communications. Current optical devices manipulate light via interactions due to their refractive indices and other controllable characteristics. Refraction, reflection, absorption, and diffraction are mechanisms by which light can be controlled and modified to achieve whatever outcome we want, to focus light into a specific area that is a lens or to absorb the light and harvest its energy that is a solar cell [3]. These days as technology advances and devices are getting smaller and smaller, the demand for optical devices to become smaller and more efficient continues to rise as consumer based optical products need to be lightweight and portable.

Metamaterials are a state-of-the-art technology that is usually composed of periodic subwavelength metal and dielectric structures. These are usually formed into a type of meta-atoms that act in the same way as conventional atoms do with regard to optical manipulation, but with the added bonus of being fully under control of the designer in terms of the materials they are made of and the size and structure of the atoms. They work by resonant coupling to electric, magnetic, or both components of the incident lights magnetic field to result in effective responses that cannot be found in nature [2-9]. As we develop our knowledge about and skill of fabricating metamaterial devices, we open the door to new and exciting applications and the possibility of optical responses that could not have been realised before, such as electromagnetic invisibility [10].

In this review paper, we will concentrate on two specific uses of metamaterials. Firstly, as perfect absorbers and secondly as perfect reflectors, the structures and mechanisms for unity or zero absorption, respectively, and their development over the last few years will be discussed. One of the main obstacles in metal based metamaterial development is that as frequencies reach the resonant frequency, surface plasmons are excited and saturate the magnetic response, rendering metal based metamaterials useless at higher frequencies. Ohmic losses are also a stumbling block as metals are lossy and heat up during use. Novel and ingenious methods of overcoming these problems will be discussed here. 


\section{Perfect Absorbers}

Perfect absorbers have been a hot topic in metamaterials for lots of years. The first realisation of a perfect absorber was published over 30 years ago [11] and ongoing research is developing, improving, and finding new and innovative ways to demonstrate and fabricate perfect absorbers.

The simplest model for a perfect absorber would be a homogeneous absorbing medium in contact with air. The absorbing medium should have both absorption, the imaginary part of refractive index, and wave impedance of unity to match the impedance of air. If there is an impedance mismatch, there will always be reflection which is obviously undesirable for a perfect absorber. The wave impedance depends on the ratio between electric permittivity $\varepsilon(\omega)$ and magnetic permeability $\mu(\omega)$. Most materials have a permittivity larger than unity, so we need materials with an equivalent larger than unity permeability too.

In short, for a perfect absorber with unity absorption $A$ we need to simultaneously minimise the transmission $T$ and reflectivity $R$; that is, $A=1-R-T$. Transmission is easily minimised by utilising a metallic back plate. The reflectivity of an incident electromagnetic wave normal to a metamaterial absorber is given by

$$
R=\left|\frac{Z(\omega)-Z_{0}(\omega)}{Z(\omega)+Z_{0}(\omega)}\right|^{2},
$$

where $Z(\omega)=\sqrt{\mu(\omega) \mu_{0} / \varepsilon(\omega) \varepsilon_{0}}$ is the impedance of the metamaterial absorber and $Z_{0}=\sqrt{\mu_{0} / \varepsilon_{0}}$ is the impedance of free space. For reflectivity to be zero, the impedance match condition, $Z(\omega)=Z_{0}(\omega)$, must be met. Therefore, the condition $\mu(\omega)=\varepsilon(\omega)$ must be satisfied. Since there are no natural magnetic materials in the optical regime, that is to say, $\mu=1$ for all materials, a metamaterial with a nonunity permeability is required. Metamaterials allow us to tune the electric permittivity $\varepsilon(\omega)$ and magnetic permeability $\mu(\omega)$ simultaneously and can be designed to allow us to reach perfect absorption at a certain frequency by impedance matching with free space.

Original designs relied on simple gratings [11-17] for their working mechanism. These gratings induced first-order and second-order air gap mode resonances which interact with the incident light to perfectly absorb it. The main drawback of these original designs was the lack of tunability [15], polarisation dependence [3], and narrow bandwidth [7], and they were extremely dependent on the angle of the incident light [3-7, 11-17]. Many new devices and mechanisms have been proposed, presented, and improved on since then; we will discuss them here.

2.1. Subwavelength Hole Array. A huge drawback of using gratings as a tool for absorption is their reliance on delocalised surface excitations [3]. These local surface excitations are extremely sensitive to the angle of incidence $[3,16]$; therefore this kind of grating structure cannot be utilised in practical applications such as microscale lighting and photovoltaic cells, where a broader range of angles are required.
To overcome the angle sensitivity of local surface excitations, Teperik et al. [3] produced an effect that relies on the excitation of localised plasmons by using a subwavelength hole array. They described how, by using localised plasmon excitation, they can successfully achieve the desired omnidirectional absorption effect needed for the applications previously mentioned. Additionally, by focusing on localised plasmon excitations, the absorption effect from the subwavelength hole array is also polarisation independent. The localised plasmons couple to the external light and decay into the metal, producing the required effect; this will be described below.

A nanoporous metal surface was fabricated by electrochemical deposition of gold over a monolayer of latex spheres supported on a gold substrate. Later the latex spheres are removed to leave empty spherical voids inside a thickness of gold. Details about this procedure can be found in [18].

Under the conditions that diffraction and polarisation conversion are suppressed, an optically thick sample that exhibits a surface resonance can become a perfect absorber if it is designed so that the radiative decay rate of the resonance is equal to the dissipation rate. If these requirements are met, the reflectivity at a frequency $\omega$ becomes

$$
R=\frac{\left(\omega-\omega_{0}\right)^{2}}{\left(\omega-\omega_{0}\right)^{2}+\Gamma^{2} / 4},
$$

where $\Gamma$ and $\omega_{0}$ are the resonance width and frequency, respectively. Since the goal is zero reflectivity, it is evident that this equation will be zero and therefore reflectivity will be zero at a frequency equal to the resonant frequency, that is, when $\omega=\omega_{0}[3]$.

A sketch and scanning electron microscope image of the fabricated surface is show in Figure 1. As the fabrication is not perfect, the top of the aperture is not completely closed, so some red shift is observed due to the leaking of the void plasmons. This redshift is paired with a smaller radiative decay rate, so at a specific thickness the rate equipartition condition is fulfilled again and the absorption becomes close to $100 \%$.

Without the existence of surface plasmons the absorption effect described here extends to incident angles of over $60^{\circ}$ for any azimuthal angle as seen in Figure 2. For angles of more than $40^{\circ}$ surface plasmons in the flat metal interface have an obvious effect and as a result produce a nonperfect absorption. This problem was overcome by filling the voids with a dielectric medium as to modify the void plasmons energy into a region where there is no interaction with delocalised surface plasmons.

2.2. Flat Nanostructured Surface. One of the most popular and well researched methods of producing a perfect absorber is by using a nanostructured surface. Several groups including Landy et al. [19, 20] proposed metal-dielectric composite metamaterial absorbers that are thin and polarisation independent and have a wide angle of absorption. Efforts have been to improve these designs so that they also include omnidirectionality $[1,19-23]$; that is, have a wide angle of absorption for any azimuthal angle. 

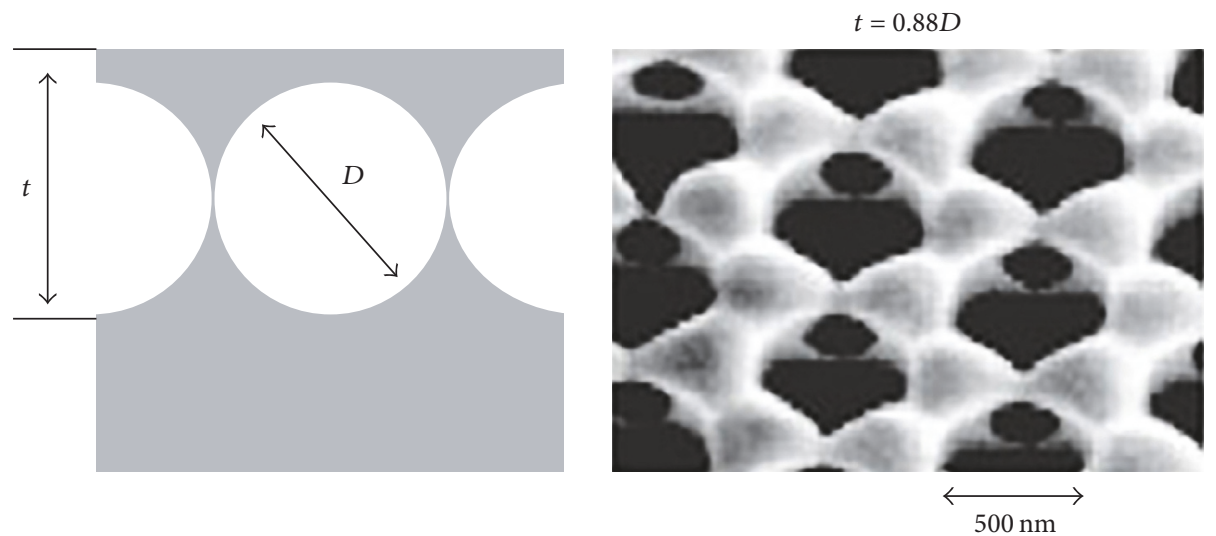

FIGURE 1: Sketch and scanning electron microscope image of the mesoporous gold surfaces used, consisting of a layer of close packed voids of diameter $D=500 \mathrm{~nm}$ covers with gold to a thickness $t$ [3].

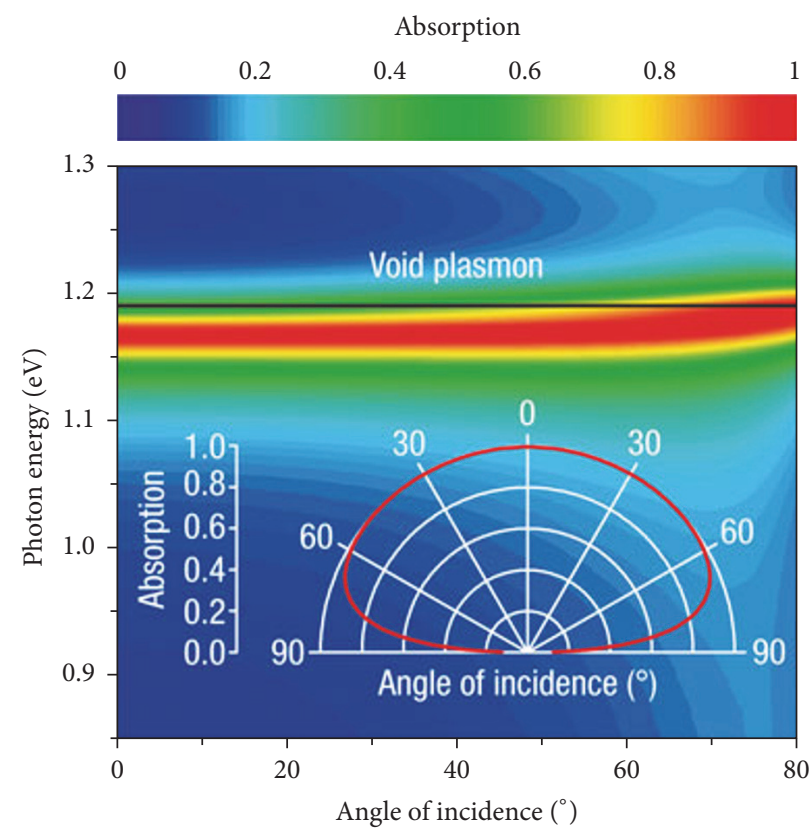

(a)

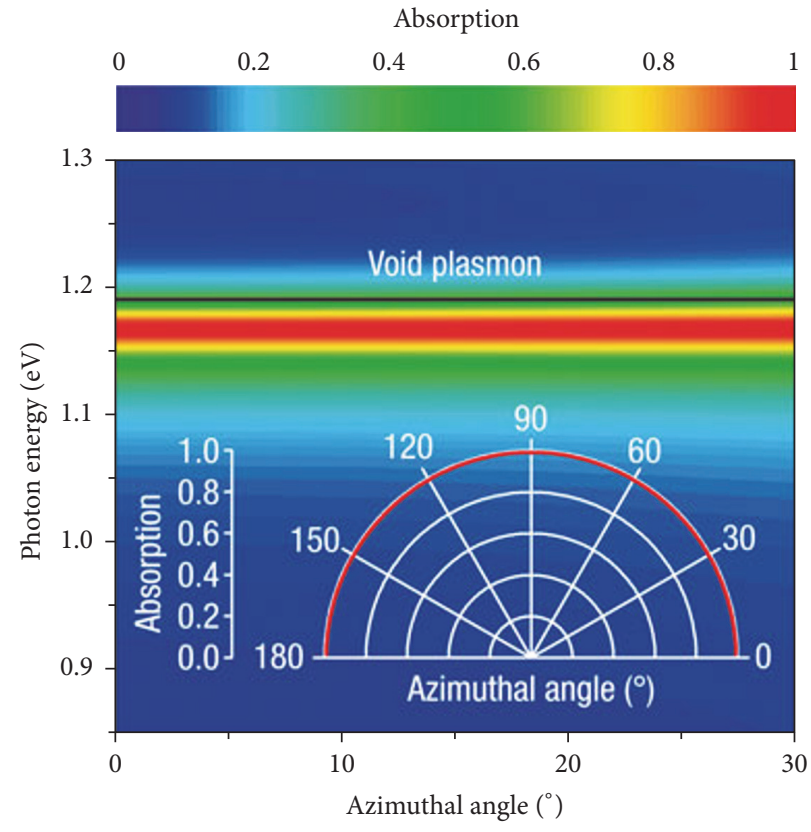

(b)

FIGURE 2: (a) Calculated incidence-angle dependence of absorption by a layer of $500 \mathrm{~nm}$ close packed silica filled inclusions buried in gold. (b) Under the same conditions as (a), there is azimuthal dependence of absorption for $20^{\circ}$ off-normal incidence [3].

Creating an absorber consisting of a polymer separation layer between a metallic film and a cut wire array it is possible to achieve up to $99.9 \%$ absorption [21] when the dimensions are properly designed. In the cut wire design three dimensions are adjustable, the thickness of the polymer layer, the width of the cut wire, and the length of the cut wire. The thickness of the metallic film is chosen to ensure that the transmission of light is zero; therefore reflection is the only mechanism for absorption.

To understand the mechanism behind the absorption simulations were done to analyse where the resonances occurred. In Figure 3 it can be seen that charges with opposite signs accumulated at opposite ends of the cut wire; this excited an electric dipole resonance. This resonance in turn is coupled to its own image which oscillates on the metallic film in antiphase. Due to this a magnetic polariton is formed and induces a magnetic response, causing a dip in the spectrum [24-26]. This electric dipole resonance strength is mainly dependent on the separation between the cut wire and the metal film, that is, the thickness of the polymer [25]. It is possible to find an optimal thickness whereby the magnetic and electric responses are impedance matched to the free space and the reflection is reduced to less than $0.1 \%$ at resonant frequency.

By considering a circuit driven by the magnetic polariton resonance, it can be deduced that the resonant frequency is 


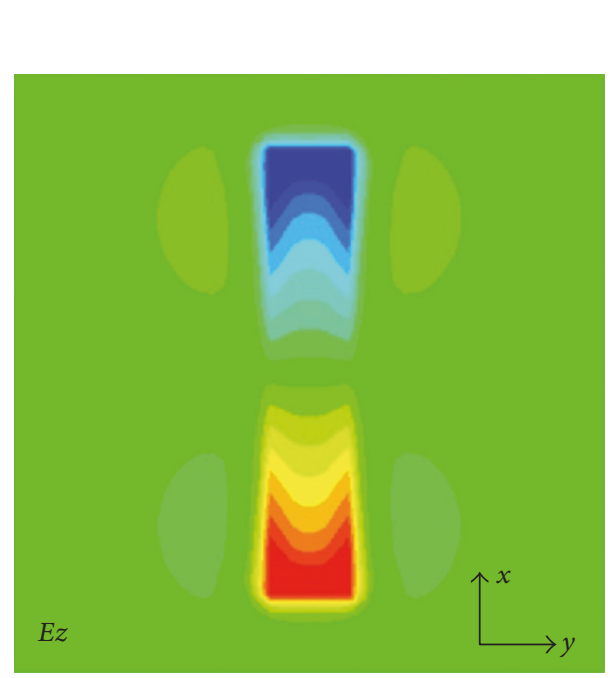

(a)

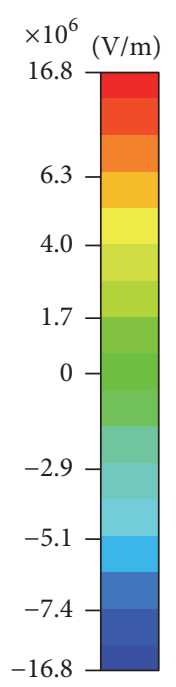

FIgURE 3: ((a) and (b)) Distribution of the $z$ component electric field for resonance on the cut wire and metallic film, respectively [21]. independent of the thickness of the polymer [27] and can be given by

$$
f_{m}=\frac{1}{2 \pi \sqrt{L C / 2}} \sim \frac{1}{l}
$$

where $L$ is the inductance, $C$ is the capacitance, and $l$ is the length of the cut wire. From this we can clearly see that the resonant frequency is solely dependent on the length of the cut wire.

By replacing cut wires with crosses, omnidirectional absorption can be achieved for both TE and TM polarisation. Polarisation independence can be easily understood since any incoming plane wave can be decomposed into two components with magnetic field components in the $x-z$ or $y-z$ plane; hence each component can excite the equivalent magnetic polariton. However, for TE polarisation at an angle of incidence above $60^{\circ}$, the $y$ component of the incident magnetic field rapidly tends to zero; consequently it is unable to excite the magnetic polariton efficiently and the absorption rate becomes low. A schematic of these designs can be seen in Figure 4.

Another goal for perfect absorbers is to increase the working bandwidth of absorption. This can be done by laying the structures on top of each other as shown in Figure 5 using layer-by-layer fabrication techniques [28-31]. Since the resonant frequency is only dependent on the length of the cross, it is possible to tune the lengths to be slightly different in different layers and ensure that the resonant frequencies of the inducted magnetic polaritons are close to each other. The stacked structures each excite their respective magnetic polaritons and since the resonant peaks are closely positioned there becomes a hybridisation of the magnetic polaritons. As a result, the bandwidth is effectively enhanced. In [21], a stack of 3 structures was investigated and a $1.03 \mathrm{THz}$ frequency band with more than $97 \%$ absorption was achieved. Finally, the thickness of the whole structure was less than $\lambda / 25$ which is much smaller than previously reported methods [3] using metal-dielectric interfaces.

2.3. Two-Dimensional Nanostructure. Rather than cut wire or cross shaped surfaces, perfect absorbers made from twodimensional disk arrays have also been thoroughly studied $[32,33]$. In this type of device, the structure is extremely similar to the previous example of nanostructured surfaces, a dielectric spacer sandwiched between a metal mirror and a structured top layer. With the main difference here being the top layer, rather than flat cut wire or cross shapes, an array of two-dimensional metallic nanorods are fabricated on top of the dielectric layer as seen in Figure 6.

In a study by Liu et al. [32] a gold disk array is structured on top of an $\mathrm{MgF}_{2}$ dielectric spacer and a gold mirror. Again, the gold mirror acts to eliminate all transmittance across the near infrared frequency regime. For a perfect absorber, $A=$ $1-T-R$. Since the transmission is zero due to the mirror, $T$ is zero. Therefore, to achieve a perfect absorber, $R$ has to be minimised.

Current distributions were studied to find the mechanism for the absorption in the device, and unsurprisingly it is due to the same mechanism as the cut wire and cross shaped nanosurfaces $[3,21]$, that is, due to antiparallel currents because of being excited in the metallic layers, inducing a magnetic moment inside the dielectric layer that can strongly interact with the magnetic field of the incident light [32]. At resonance, the localised electromagnetic field between the layers is enhanced greatly $[26,32,34,35]$ and therefore electromagnetic energy can be confined inside the dielectric spacer, minimising the reflectance. Using this method, a perfect absorber was realised with almost zero reflectance $(R=0.28 \%)[33]$.

An advantage of using an array of $2 \mathrm{D}$ cylindrical nanorods on the top surface is that it is compatible with all current nanofabrication techniques such as electron beam 


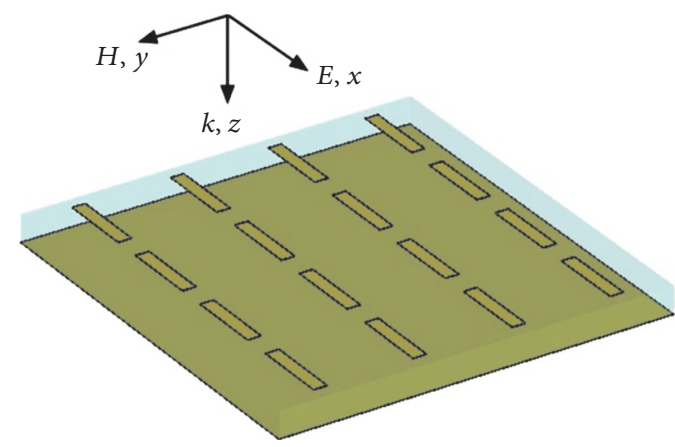

(a)

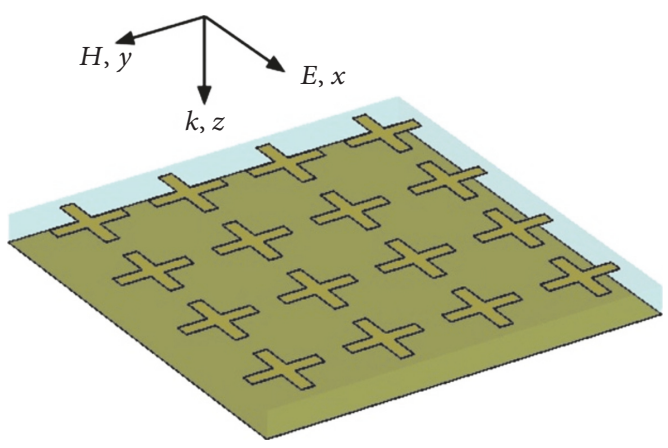

(c)

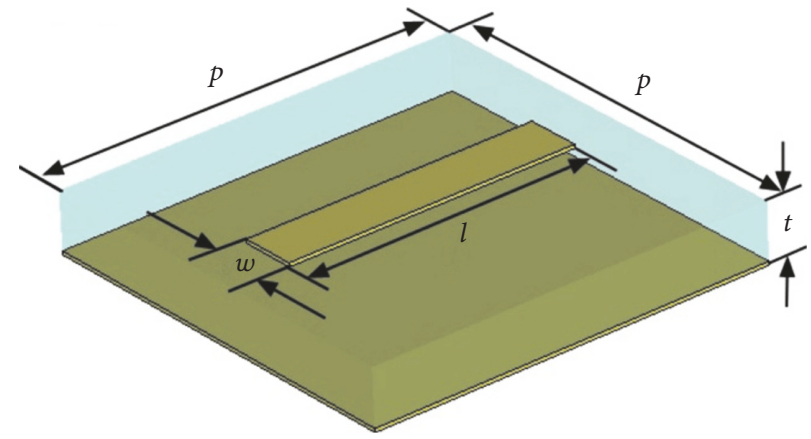

(b)

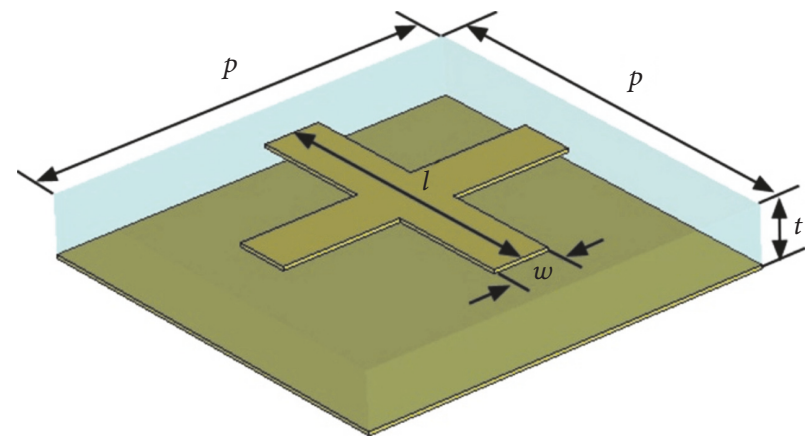

(d)

FIGURE 4: Schematic diagrams of the THz absorbers consisting of a metallic film, a polymer separation layer, and (a) a cut wire array and (c) a cross array. (b) and (d) are unit cells of the two absorbers [21].

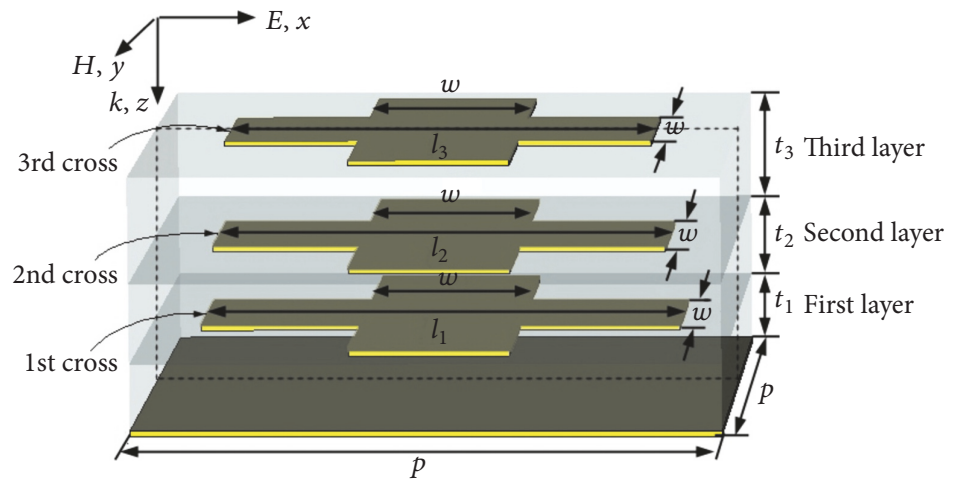

FIGURE 5: Schematic diagram of a 3-layer cross structure with the geometrical parameters of each layer marked [21].

lithography [36], interference lithography [37], nanosphere lithography [38], and focused ion beam writing [39]. This could easy lead to the possible manufacture of ultrasensitive plasmonic detectors.

2.4. Microcavity. Microcavities have been devised in all kinds of shapes, ranging from simple gratings and rods to more intricate designs $[40,41]$. They have been shown to be polarisation dependent, polarisation independent, and narrowband and broadband depending on the dimensions and design. Here a few select designs will be discussed and compared for simplicity.
2.4.1. "Bottle-Like" Narrowband Absorber. As illustrated in Figure 7, the bottle-like absorber is made up of an array of $\mathrm{SiO}_{2}$ strips embedded in gold with small air gaps at the top of the strips. Gapped nanoparticles have been proved to be able to concentrate light and therefore as the gap resonance the electric field inside the gap can be enhanced greatly. The origin of the absorption and the location of the resonance enhanced field are in the gap.

The width and height of the dielectric strips can be tuned as desired to produce the desired spectra. It was shown that over all range of widths and heights the spectra always remain over $97 \%$ absorption. This is only true to TM polarised light 


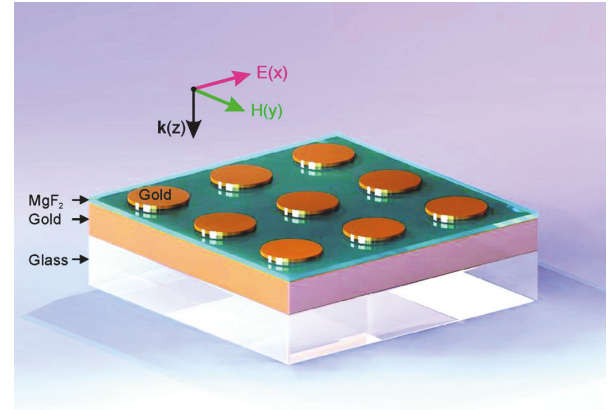

FIGURE 6: Schematic diagram of the two-dimensional structured perfect absorber [32].

only, as shown in Figure 8; for TE polarised light almost all the light is reflected ( $<2 \%$ absorption). This microcavity absorber is also extremely angle independent, as long as the incident electric field is parallel to the $x z$ plane. At angles up to $65^{\circ}$ the absorption resides at over $80 \%$.

Electric intensity enhancement was investigated to find the mechanism for the absorption. It can be seen that absorption is at its maximum when the electric field intensity is at its maximum. The dimensions of the dielectric correspond to different resonant wavelengths to maximise the electric field enhancement in the air gap.

Although this design has lots of benefits, such as high performance, fine tenability, and strong polarisation dependence, it is fairly difficult to fabricate. Therefore, in practice more simple structures are required to allow straightforward fabrication and later scalable manufacture.

2.4.2. "Cup-Like" Narrowband Absorber. The design of the cup-like narrowband absorber can be seen in Figure 9. It is made up of nanoscale grooves covered in a layer of dielectric. The width and the depth of the grooves can be tuned, as can the thickness of the dielectric layer and the period of the grooves.

The mechanism of the absorption can provide an almost perfect absorber at resonant wavelength. This is due to the destructive interference between light reflected from the top surface and the surface inside the grooves. The resonant frequencies are found to be adjustable by varying the thickness of the dielectric layer; meanwhile as the thickness of the dielectric layer or the depth of the grooves increases, the absorption peaks are redshifted.

Again, this type of absorber is highly polarisation sensitive at no normal incidences as in the previous case. In this case, TM polarised light has absorption of over $85 \%$ for an incident angle of up to $50^{\circ}$ and the absorption effectively disappears under TE polarised light. For both of the aforementioned microcavity perfect absorbers it is possible to change the refractive index of the dielectric material to cause a red shift in the absorption spectra without changing the overall absorption. It is reported that using these two types of perfect absorber that it is possible to perfectly absorb any polarised electromagnetic wave with wavelengths ranging from $700 \mathrm{~nm}$ to $2300 \mathrm{~nm}$, red to infrared light.

2.5. Periodic Resonator. Periodic resonators consist of an electric-field-couple-LC resonator and a metallic backplate that are separated by a thin dielectric layer. These ultrathin devices can be arranged in a periodic manner to create perfect absorbers over a range of working frequencies [42-47]. The electric-field-couple-LC resonators are scaled and arranged in a way so as to regulate the effective capacitance and inductance of the overall structure to realise the maximum possible resonance.

A schematic of the device can be seen in Figure 10. The top layer of electric-field-couple-LC resonators is responsible for the electric resonance which couples with the metallic back plate to create a magnetic field that drives the current in the resonator. The centred capacitor and inductors only contribute when the incident electric field is perpendicular to the capacitor. By structuring two sizes of these single resonators into an alternating pattern a dual band perfect absorber is produced. The electric and magnetic resonances are activated individually which confines the electromagnetic power into the cell of the resonator [43].

The resonance between two cells is very weak and the absorption is independent among each element, so it is safe to assume that making arrays of different scale resonators will produce peaks due to each size independently. Thus, a triple band resonator consisting of 3 scales was fabricated and investigated [44]. As expected three distinct absorption peaks were observed in the $\mathrm{GHz}$ frequency, all of more than $93 \%$ absorption in frequency bands over wide angles of incident waves for both TE and TM polarisation.

Another design of a triple band absorber is shown in Figure 11. This design [44] has fourfold symmetry and the same mechanisms are at work as in the previous study [43]. The copper resonator is responsible for the electric field response and the coupling between the top and bottom layers create the antiparallel surface current that excites the magnetic response as shown in Figure 12. Due to the symmetry, the response to both TE and TM polarisation is almost identical, and the absorption is also strong at most incident angles.

2.6. Nanostructured Metal-Dielectric-Metal Stack. In general, reducing metallic losses [48-50] is one of the most sought after goals in nanophotonics, so designing perfect absorbers that are based on only lossless dielectric materials and noble metals is a highly active area of research. Here we will discuss a few designs and mechanisms for creating a perfect absorber from lossless materials.

At lower frequencies such as infrared, noble metals like copper, silver, and gold are great reflectors but display unwanted plasmon resonances at visible frequencies [5]. By creating a subwavelength noble metal structured surface, localised and delocalised surface plasmon resonances can be utilised to realise high absorption on usually reflective surfaces.

An absorber consisting of a thin $(260 \mathrm{~nm})$ three-layer metal-insulator-metal film was fabricated [5]. Only the top 


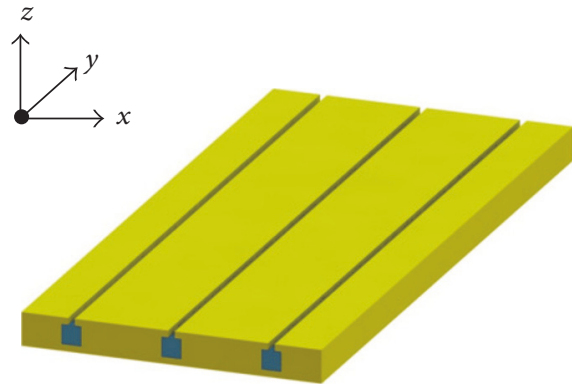

(a)

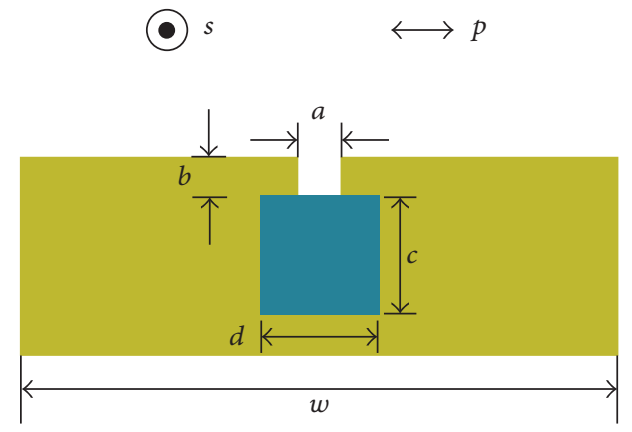

(b)

FIGURE 7: (a) Schematic of the bottle-like absorber: the yellow region is gold and blue regions are the dielectric. (b) Cross section of a unit cell, the width, and depth of the air gap as represented by $a$ and $b$, respectively [42].

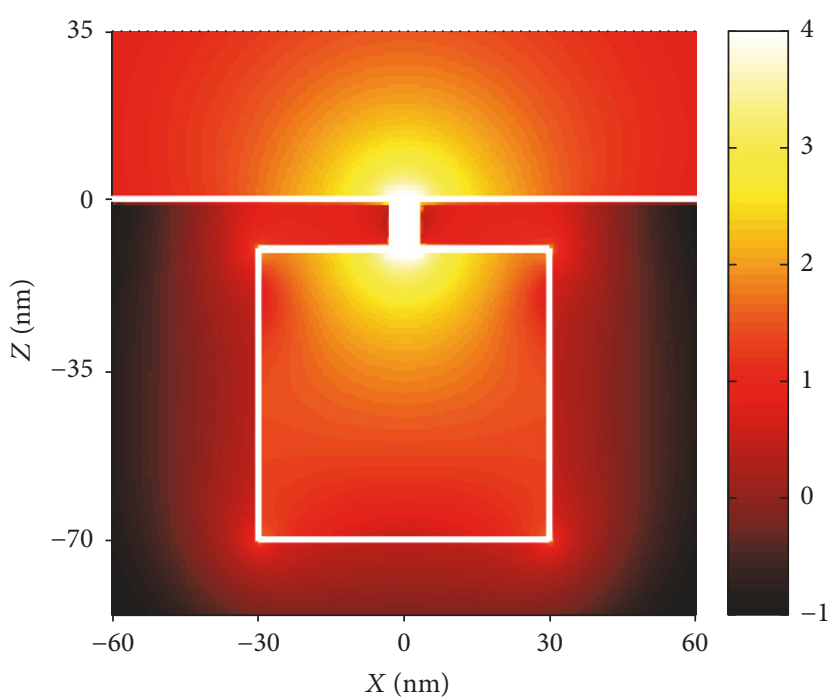

(a)

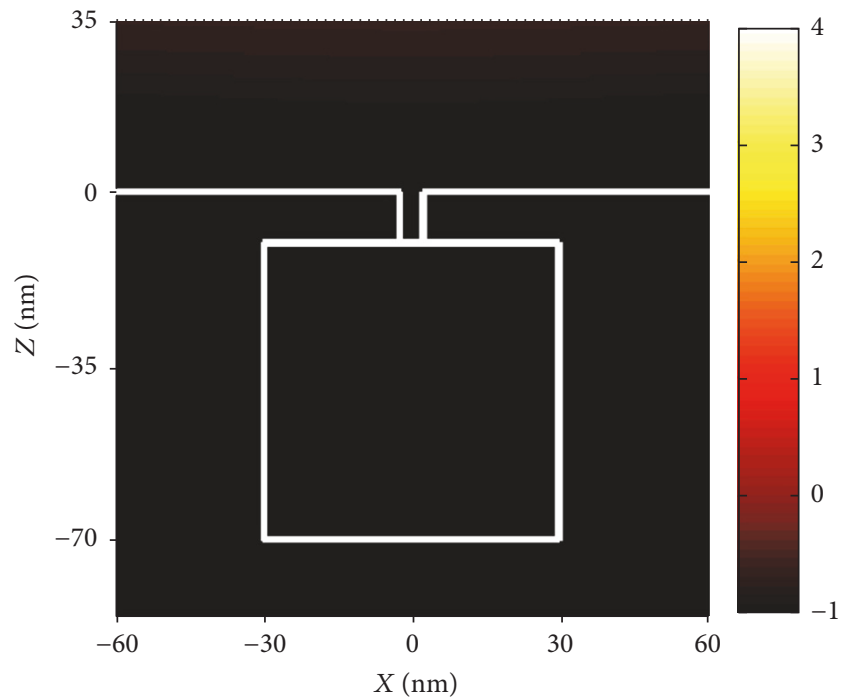

(b)

FIgURE 8: Comparison between the electric intensity enhancement under (a) TM polarised light and (b) TE polarised light [42].

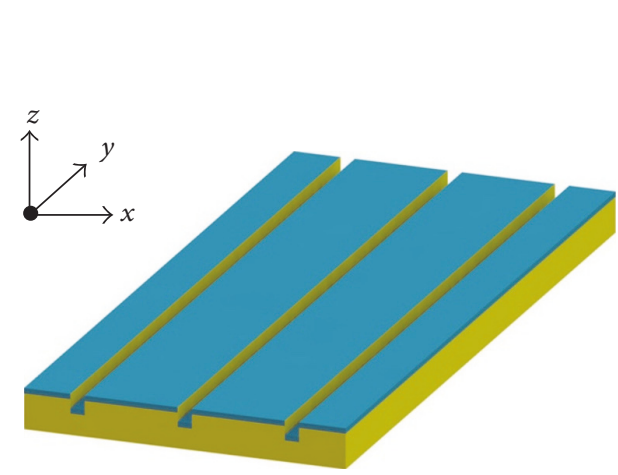

(a)
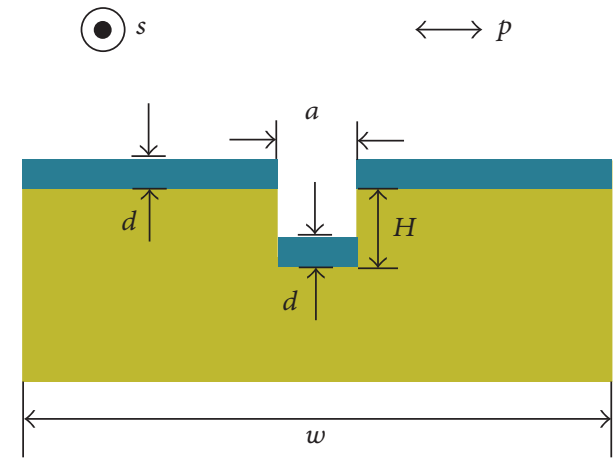

(b)

Figure 9: (a) Schematic diagram of the cup-like perfect absorber: the yellow region is gold and the blue regions are dielectric. (b) Cross section of a unit cell [42]. 


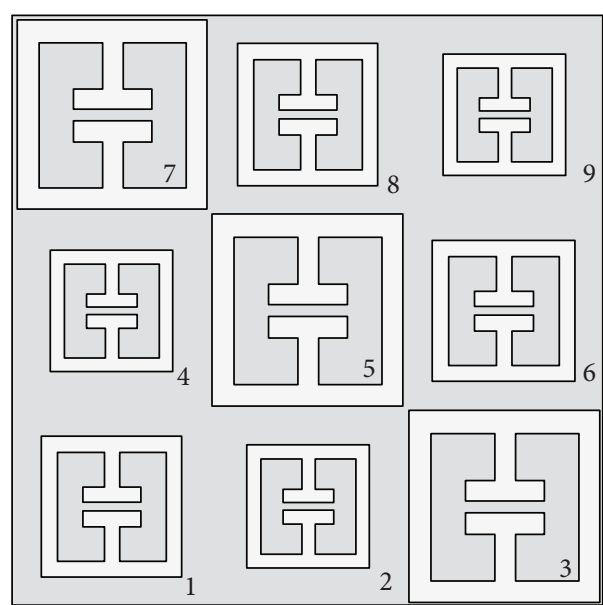

(a)

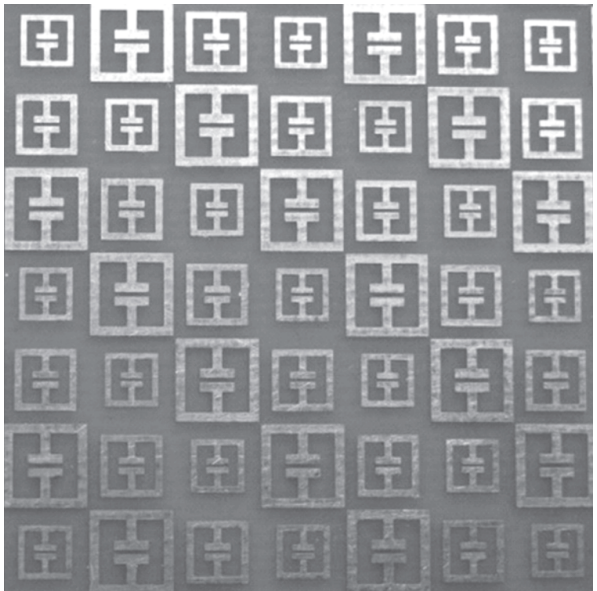

(b)

FIGURE 10: (a) Schematic of the periodic resonator. (b) The fabricated structure [44].

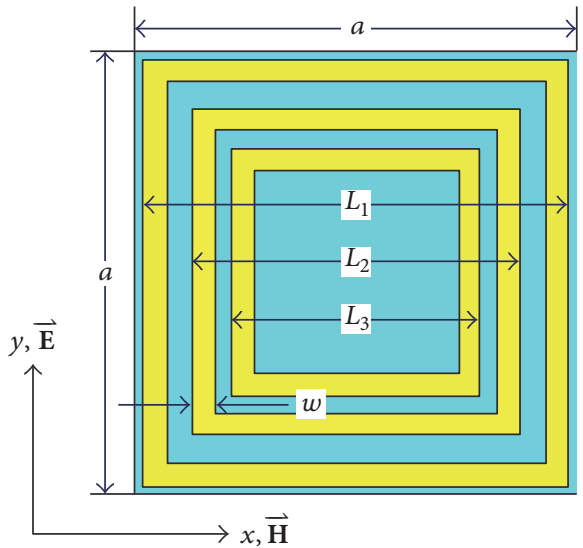

(a)

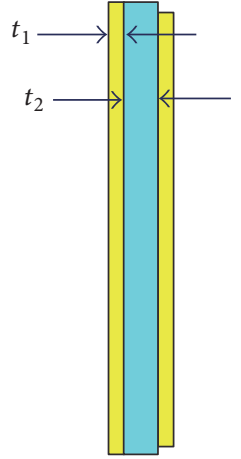

(b)

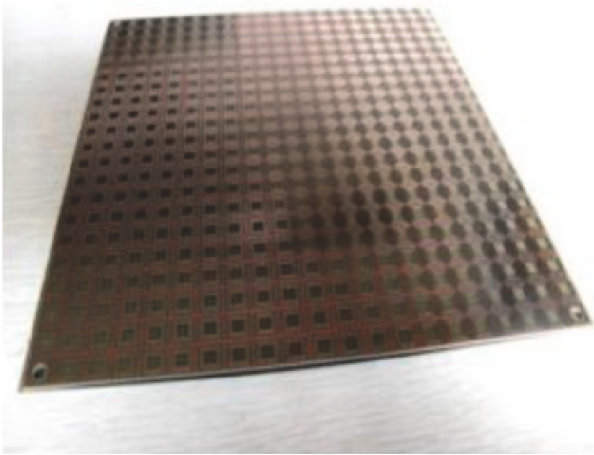

(c)

FIGURE 11: Schematic structure of the triple band microwave absorber (a) from the front and (b) the side [43].

layer was patterned. Two patterns were investigated, a metal strip array and a trapezoid array as can be seen in Figure 13. The pattern was repeated over the $x$-axis to give a periodic pattern. Again, the localised surface plasmon resonances could be identified from the absorption in the spectra, and as always they produce narrowband resonances which are dependent on the width of the pattern. As the patterns width was increased, the resonances were red shifted. When the electric field of the incident light is parallel to the wire arrays, that is under TE polarisation, the wire arrays act as effective plasma media. But this resonant behaviour is much weaker in the case of TM polarisation, showing strong polarisation dependence.

To remove the polarisation dependence of the periodic wire pattern, from intuition we can assume that a symmetrical shape could be useful. By arranging the trapezoidal wires into an array of crosses this feat was accomplished [5]. Trapezoidal shaped cross arrays were able to produce a broad absorption spectrum of over $70 \%$ that ranged from 400 to $700 \mathrm{~nm}$. This is extremely significant as it is in the range of visible light. By calculating the electric response, it was shown that different parts of the trapezoidal wire were responsible for exciting of different resonances at different frequencies.

2.6.1. Ultrathin Metal Layer. To continue the development of layered perfect absorbers, a method based on one ultrathin layer of chromium between two silica layers was devised $[3,51]$. The thin metal layer is no longer a noble metal and no longer requires any structuring. By using the impedance transformation method [52], chromium was chosen as the refractory metal as it most closely matches the ideal permittivity in the visible and near infrared range [53-55]. The whole assembly is placed on a gold substrate which acts only as a mirror to give zero transmission. A schematic of the absorber can be seen in Figure 14. Absorption of over 90\% over the wavelength range of $0.4-1.4 \mu \mathrm{m}$ was shown, with angle and polarisation independence.

By investigating the electric field distribution under normal incidence shown in Figure 15, it was shown that 


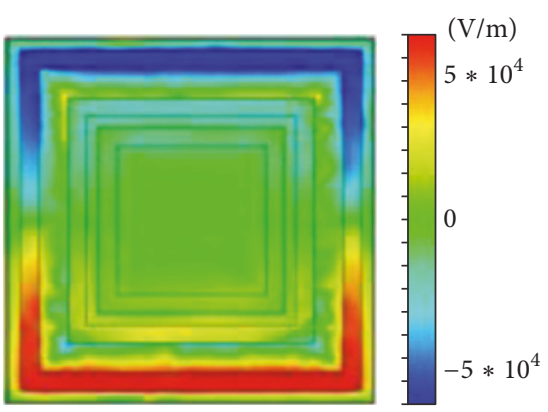

(a)

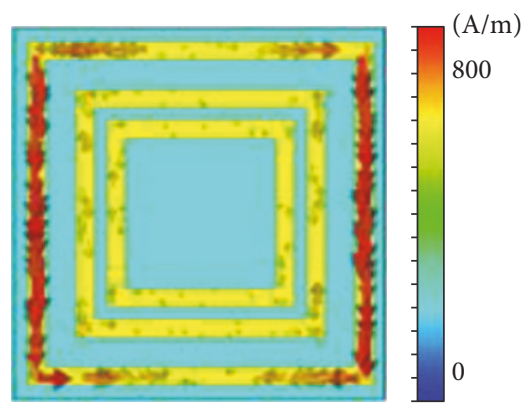

(d)

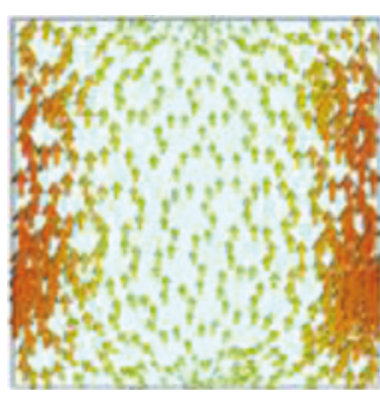

(g)

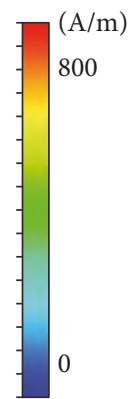

0

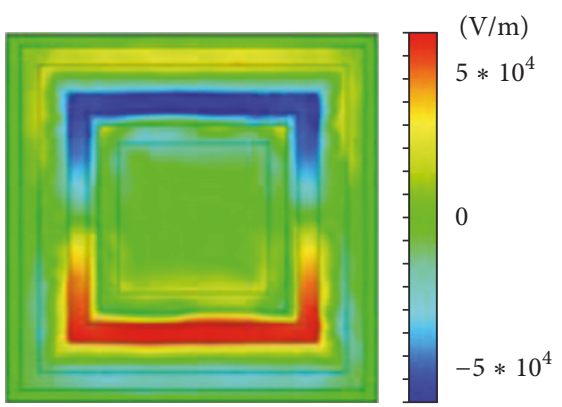

(b)

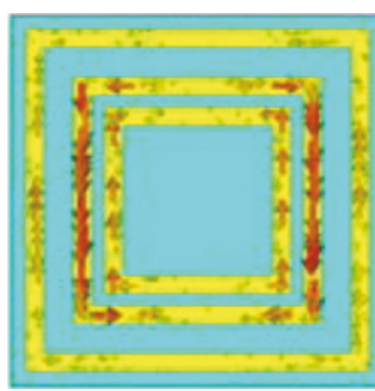

(e)

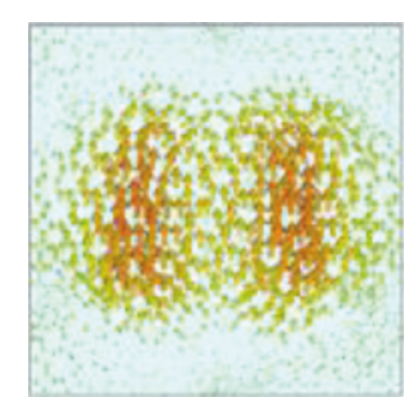

(h)

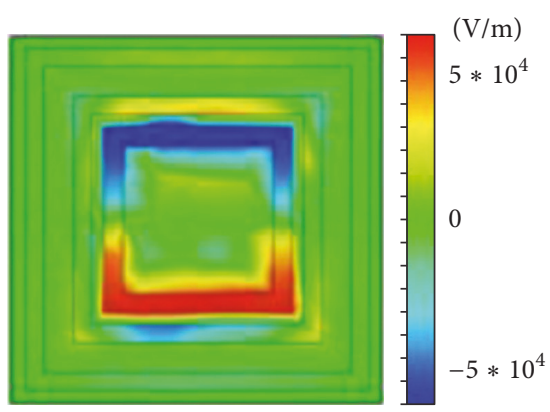

(c)
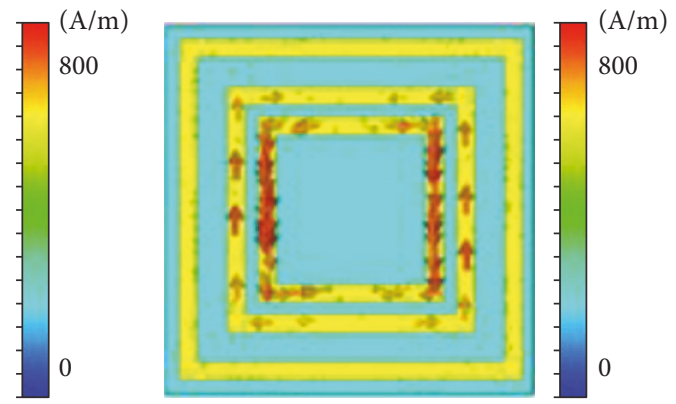

(f)
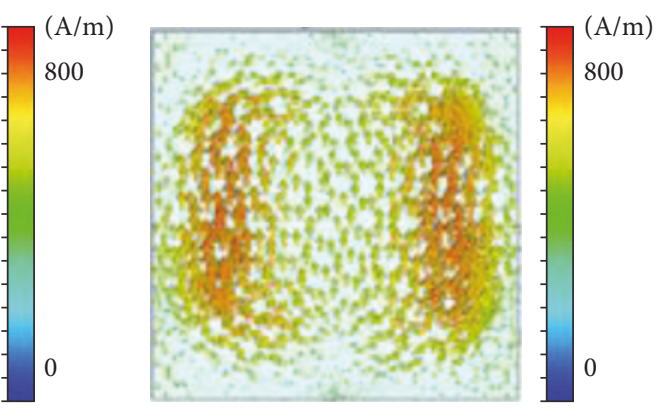

(i)

FIGURE 12: Distribution of normal electric fields and (d)-(i) surface current distributions for the three peaks of absorption. (d)-(f) Surface current distributions on the top rings; (g)-(i) surface current distributions on the ground plane [43].

the Cr layer did not reflect any light, and from the timeaveraged power flow it is obvious to see that all of the light is trapped and absorbed by the thin Cr layer, not in the dielectric layers. Since refractory metals such as $\mathrm{Cr}$ have extraordinary resistance to heat and high melting points compared to noble metals such as gold and silver [56], this kind of absorber could have utility in applications such as high temperature thermal emitters in the visible and near infrared range [57, 58], solar energy harvesting [3], and thermal imaging [2].

2.6.2. Additional Grating. Although gratings were one of the first methods of creating a perfect absorber [59], it still has valid interest to this day. It can be used to enhance certain structures by introducing features and useful tuneable parameters. Dielectric gratings and waveguides have been previously studied [60] whereby the surface plasmon polaritons form a surface wave between the dielectric and metal due to the oscillation of free electrons at the interface.
In [61] rather than creating a waveguide or grating into a dielectric top layer, the grating was constructed directly onto the silver surface. This resulted in a simple absorber with a subnanometer bandwidth. The resonant wavelength of this absorber is very easily tuneable via geometrical scaling and is also ultrasensitive to the refractive index and the dielectric substrate. A schematic of the design is shown in Figure 16.

The temporal coupled-mode theory [58-62] is used to provide light on the mechanism of the absorption. Under illumination of the resonant wavelength, surface plasmon polaritons will be excited. This energy will dissipate into the metal as resistive heat while the remaining part is radiated into the free space. These damping rates can be varied by altering the size of the grating. Finally, the resistive damping rate can be obtained from

$$
\gamma_{\text {res }}=\gamma_{\text {tot }}-\gamma_{\text {rad }}
$$




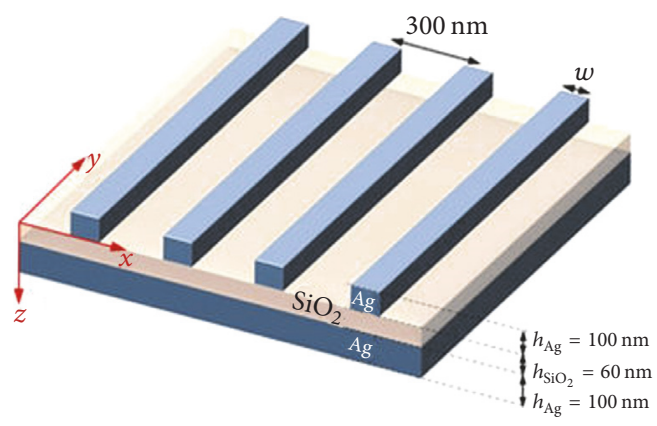

(a)
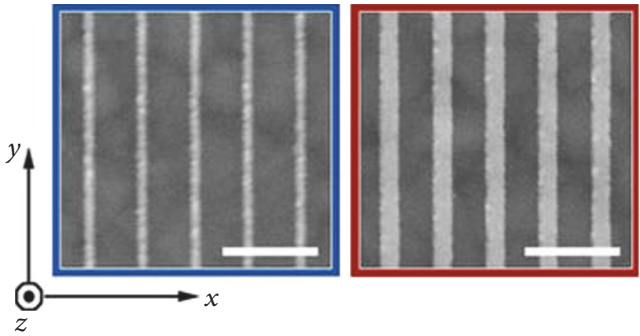

(c)

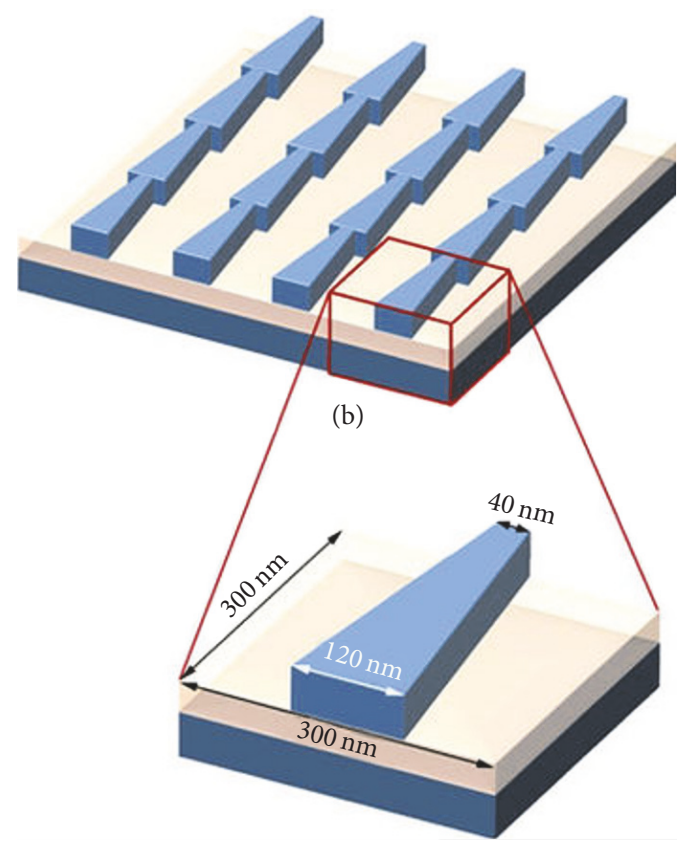

(d)

FIGURE 13: Schematic diagram of the three-layer system with (a) metal strip and (b) trapezoidal grating. (c) Electron microscope images of the fabricated devices. (d) Unit cell of the trapezoid grating [5].

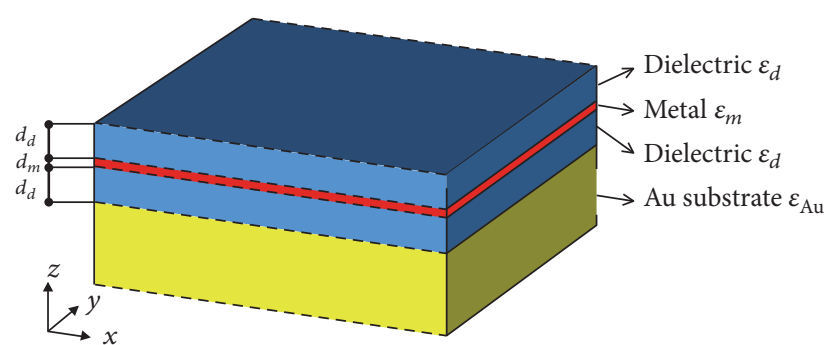

Figure 14: Schematic diagram of the broadband perfect absorber consisting of a single thin layer of metal between two dielectric layers on a gold substrate [51].

where $\gamma_{\text {tot }}$ is the total damping rate and $\gamma_{\text {res }}, \gamma_{\text {rad }}$ are the resistive damping rate and radiative damping rates, respectively. The absorbance at angular frequency $\omega$ is given by [63]

$$
A=\frac{4 \gamma_{\mathrm{rad}} \gamma_{\mathrm{res}}}{\left(\omega-\omega_{0}\right)^{2}+\gamma_{\mathrm{tot}}^{2}} .
$$

Peak absorbance is at $4 \gamma_{\text {rad }} \gamma_{\text {res }} / \gamma_{\text {tot }}^{2}$ at $\omega-\omega_{0}$. Critical coupling occurs when $\gamma_{\text {rad }}=\gamma_{\text {res }}$ and leads to unity absorption, a perfect absorber as desired.

Absorption of $100 \%$ was observed at a variety of wavelengths, which can be tuned by changing the geometrical scaling factor. Interestingly the absorber has strong resonances as different angles for different wavelengths of light. At all wavelengths, the FWHM is very small, around $0.6 \mathrm{~nm}$, which would be ideal for practical use as a plasmonic sensor.

Meanwhile, Wu et al. [64] worked on a similar structure for infrared frequencies, but rather than structuring directly onto the top metal layer, they proposed a multilayered metaldielectric-metal structure, with the top layer being structured into a grating.

To understand the absorption mechanism, again the electric field intensity distribution at the resonant wavelength was studied and is shown in Figure 17. Below the slits there are large electric field intensity enhancements and concentration inside the dielectric spacer. This can be attributed to the guided mode resonance. As the dimensions of the grating change, the location of the resonant wavelengths also changes in order to satisfy the phase matching condition, but for all wavelengths the absorption peak is still quite high (more than $90 \%$ ) for angles of incidence up to $34^{\circ}$, and this shift does not go outside of the width of the absorption peak.

To enhance this single band perfect absorber [65], it was modified to become a multiband absorber by simply stacking more layers of metal and dielectric of different thicknesses on top of the existing structure, Figure 18. From the electric field intensity distribution, it can be seen that the power loss is greater for a certain resonant frequency in a certain layer, with standing wave profiles in the $x$ direction. This standing wave profile is a typical feature of guided mode, so the enhancement of the absorption can be attributed to the guided mode resonance, with each mode being reliant on the size of the different dialectic spacer.

The above structures work for TE polarised waves but can easily be adapted to accept TM polarisation and be polarisation independent through methods described in previous work by the same team, here [65].

In summary, dielectric nanostructures have both electric and magnetic resonances coming from Mie-like resonances 


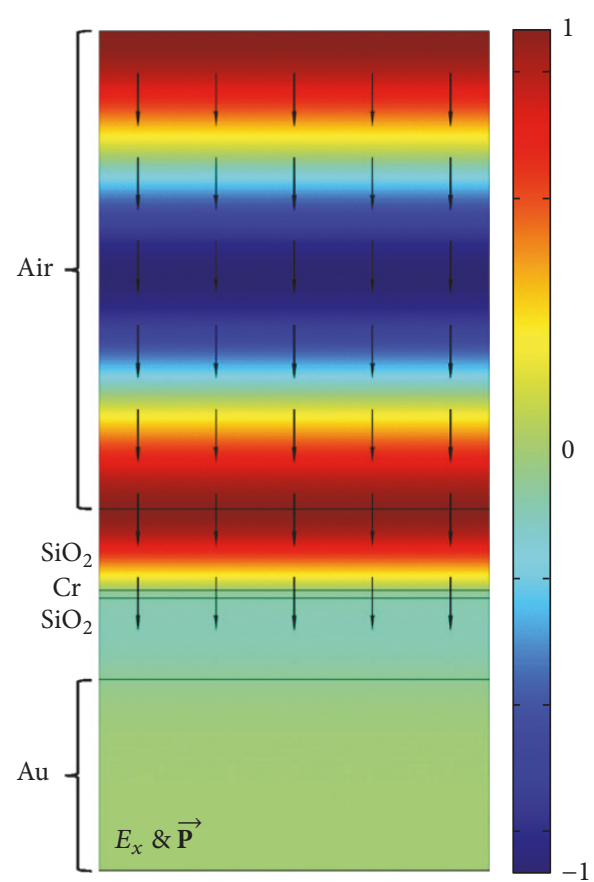

(a)

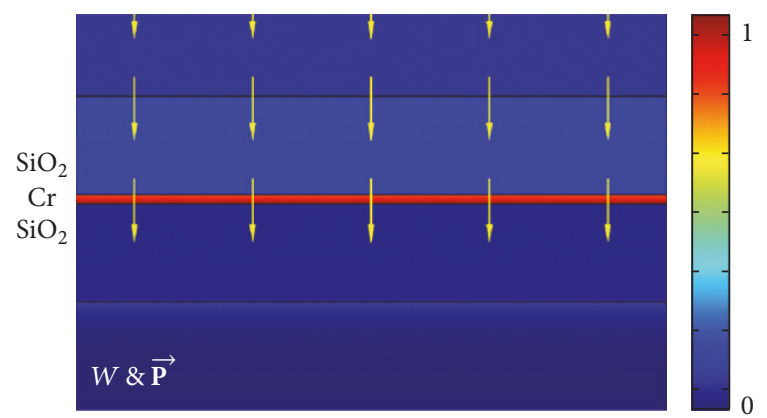

(b)

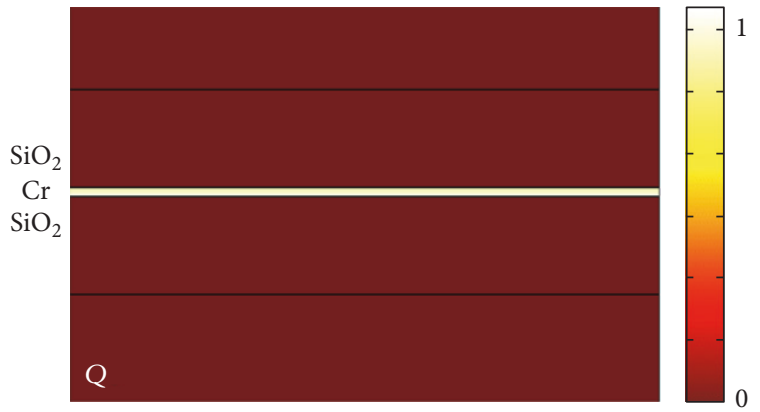

(c)

FIGURE 15: (a) The calculated electric field distribution and time-average power flow $P$, (b) time-averaged energy density distribution and power flow, and (c) heat generation density distribution under normal incidence at the wavelength of $500 \mathrm{~nm}$ [51].

without complex nanostructures. These simultaneous electric and magnetic resonances are responsible for unidirectional scattering, which we can relate to high efficiency reflection, transmission, and absorption. We can then add various nanostructures to the metal top layer to control the absorption direction and polarisation as we desire.

2.7. All Metal Subtractive Reflector. Although most of the devices presented here use a mixture of metal-dielectric multilayers so as to reduce the effects of the ohmic losses in metals [48-50], this costs extra time and money during fabrication [66-69]. So more recently all metal perfect absorbers [70] have been investigated.

Previous studies have shown how all metal surface absorbers have been achieved within nanotrenches of varying depths and broadband absorption within ultrasharp convex grooves [71, 72], but more recently $\mathrm{Ng}$ et al. [70] presented an all metal structure with individual nanostructures of equal height. This design has the added advantage of being easy and simple to fabricate via scalable patterning and etching processes or even direct printing onto metal substrates [73]. A schematic of the structure is shown in Figure 19. Three silver nanostructures were considered, a circle, square, and cross, respectively, on a bulk silver layer. By using these three shapes, absorption peaks across the whole visible spectrum are tuneable by modifying the width and shape of the metal protrusion.

Rather than relying on surface plasmon polaritons that occur at the interface between a metal and dielectric layer, these all metal structures utilise the excitation of local surface plasmon resonances instead. These allow the individual nanostructures that exhibit different colours to be in close proximity and exhibit angle independent nondiffractive colours. Narrow near perfect absorption peaks, above 95\%, were observed at fairly large incident angles as the localised surface plasmon resonances produce subtractive colours with high saturation.

The mechanism for this all metal absorber was explored by looking at the field, charge, and power distributions at resonant frequency. In Figure 20 it is clear to see that the electric field is concentrated at the top and bottom of the edge of the structure, while the magnetic field is concentrated above the top surface and to the side walls. The electromagnetic power is absorbed at the circumference at the top surface and partially at the base. This power absorption is the reason for the absorption of the incident light as we can see that no power is reflected; therefore it is completely absorbed by the structure at the resonant wavelength. Possible uses for this kind of device are as a colourant for plastic consumer products [74], in high resolution colour printing [75], and in polarisation sensitive colour printing [76].

Green colour continues to be a difficult problem, as it is difficult to produce peaks at green wavelengths using this type of perfect absorber. Black, that is absorption of all colours, is also still yet to be found using the methods reported here, but other methods such as randomly roughened and vertically tapered metal surfaces have achieved this feat $[72,77]$. 


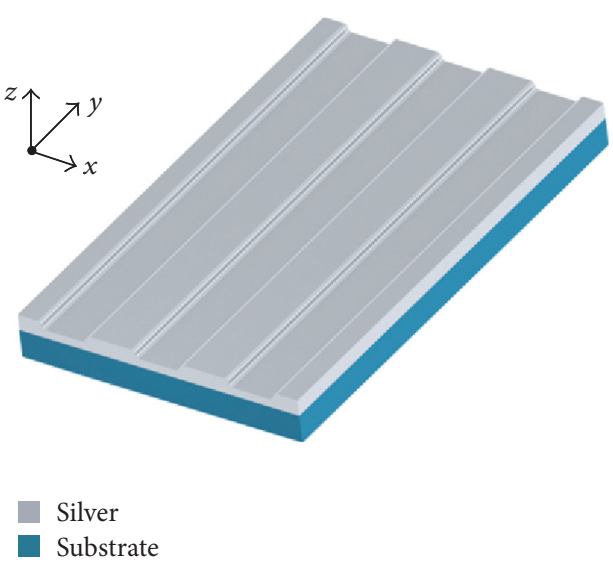

(a)

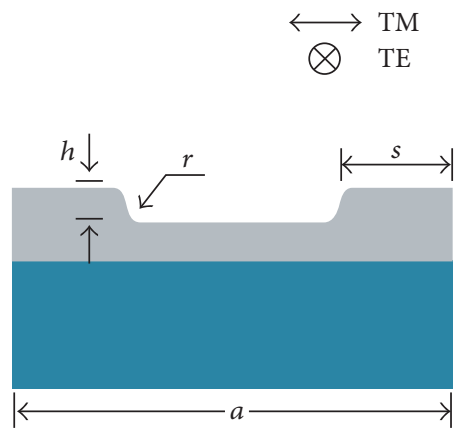

(b)

Figure 16: (a) Schematic of the 1D grating; the grey region is silver and the blue region is the substrate. (b) Cross section of a unit cell [61].

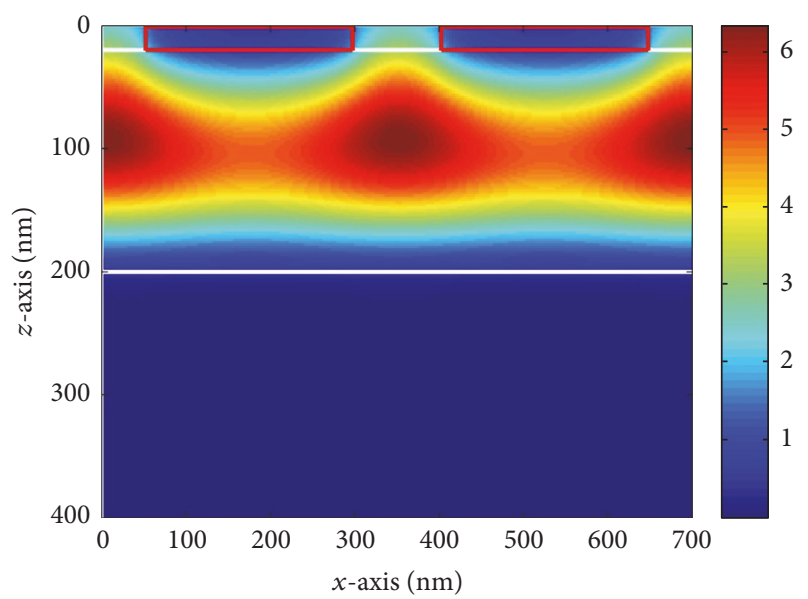

FIGURE 17: Electric field destruction of the perfect absorber [64].

\section{Perfect Reflectors}

The most common way to create a mirror is a thin layer of metal. Since metals are already almost perfect reflectors, usually with reflection over $90 \%$, they are used in most applications from astronomical telescopes to dental mirrors. The reflectance of a mirror can be calculated from the refractive index, $n$, and the extinction coefficient, $k$, using the relationship

$$
R(\%)=\frac{(n-1)^{2}+k^{2}}{(n+1)^{2}+k^{2}} \times 100 \% .
$$

The values of $n$ and $k$ are readily available for every metal over a wide range of wavelength regions. From this we can see that certain metals are only useful at certain wavelength ranges as their reflectance becomes too low at others; for the example of UV light in astronomical telescopes, silver is an unacceptable choice whereas aluminium is a great choice. The main problems with using metal films as reflectors are that in optical systems with many mirrors reflectance will degrade below 50\% quickly, and certain metals, albeit having high reflectance in the required region, will react with the local environment and will become quickly damaged.

In the case of metamaterials, one of the key problems of metallic based devices is due to ohmic dampening which results in unwanted absorption [48-50]. Moreover, as high frequencies approach the resonant plasmon frequency the excitation of the electrons in the metal saturate the magnetic response, limiting their use at these high frequency ranges. Perfect reflectors are also useful in protecting surfaces against high power radiation such as in laser cavities and although other methods can be used, perfect reflection from a single layer of metamaterial could offer many advantages such as simplifying the fabrication process and being able to produce a fast and effective paint-like coating that could be used for large area optics.

It can be shown that, to achieve unity reflectance, the real part of the impedance of the medium must be zero [78]. This condition is achieved when $\varepsilon^{\prime} / \mu^{\prime}<0$ and $\varepsilon^{\prime \prime} / \mu^{\prime}=\varepsilon^{\prime} / \mu^{\prime \prime}$, where the complex permittivity and permittivity are given by $\varepsilon^{\prime}+i \varepsilon^{\prime \prime}$ and $\mu^{\prime}+i \mu^{\prime \prime}$, respectively. The first part required that the real parts of the permittivity and permeability have opposite signs, which is easily found close to an electric or magnetic resonance, provided that the resonances are spectrally isolated. The second condition is more difficult to achieve but is satisfied in lossless materials. The imaginary part of the refractive index must also be maximised to prevent evanescent tunnelling across the device.

3.1. Metal Antenna Array. Realisation of metasurfaces with broadband optical response and high efficiency at visible wavelengths is a challenging task as usual designs of optical resonator elements, or meta-atoms, only offer limited efficiency due to ohmic losses in the metallic structures and diffraction modes inside the discrete meta-atoms [7981]. Simple plasmonic nanostructures have mostly electric dipole modes, which radiate omnidirectionally. This limits the efficiency of device which uses simple plasmonic nanostructures. For this reason, the metal on metallic substrate 


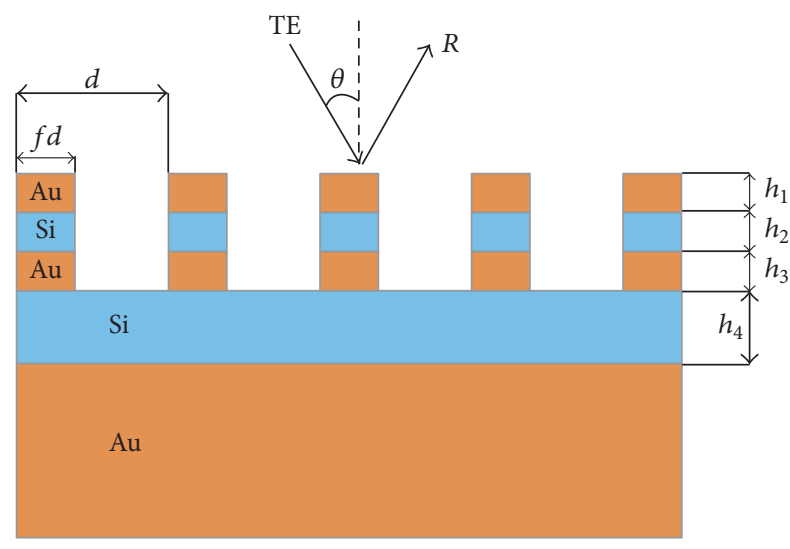

(a)

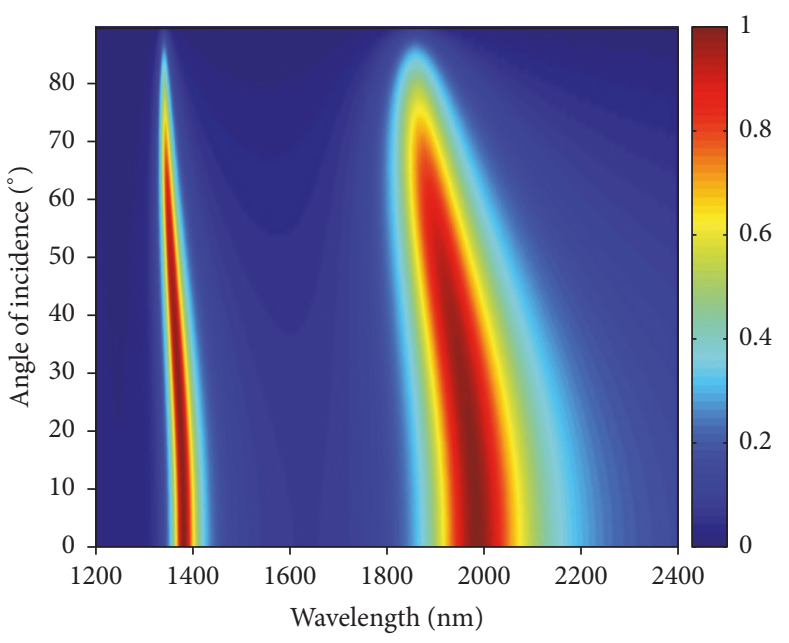

(b)

FIGURE 18: (a) Schematic of the triband perfect absorber consisting of three layers or metal and dielectric grating structure. (b) Absorbance as a function of wavelength and the angle of incidence [64].

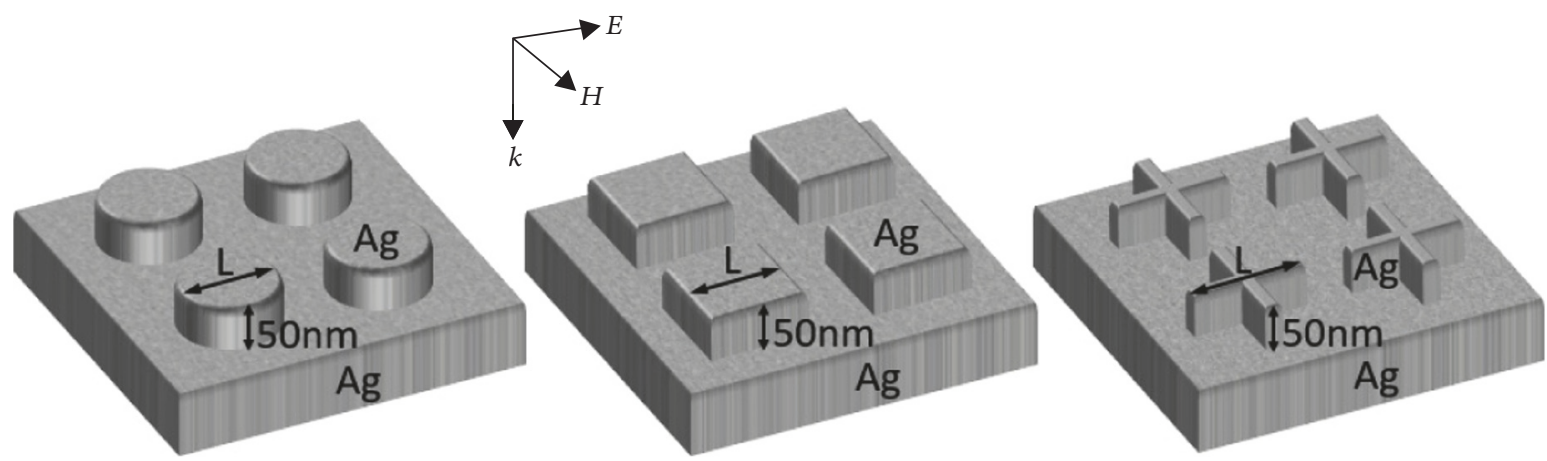

(a)
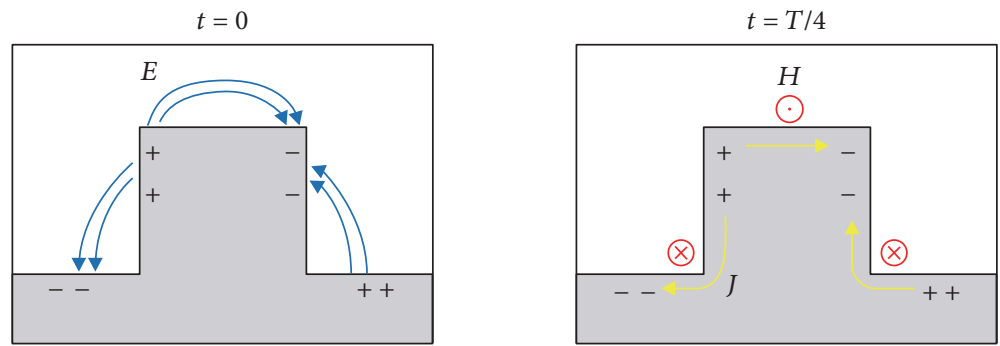

(b)

FIGURE 19: (a) Schematic diagrams of all-metallic silver structures with nanoprotrusions emerging from a bulk layer of silver for three geometries (circle, square, and cross). (b) Electric and magnetic fields at different time instances for the localised surface plasmon resonance mode [70].

and dielectric nanostructure schemes had been used for high efficiency metasurfaces. The schemes rely not only on electric resonances but also on simultaneously magnetic resonances, which make the most desirable unidirectional scattering possible.

In [82] $\mathrm{Li}$ et al. suggested a simple design to realise a broadband perfect reflector across the visible frequency range by using a single gradient meta-atom design. Rather than several discrete sized elements in the unit cell $[83,84]$, this design is basically an array of trapezoid shaped $\mathrm{Ag}$ nanorods coupled with an optically thick Ag film separated by a $\mathrm{SiO}_{2}$ spacer $[5,85]$. This kind of single atom unit cell makes fabrication much easier with high geometric accuracy [86]. The design is shown in Figure 21.

As with perfect absorbers, the absorptivity $A$ is reduced to $A=1-R-T$, where $R$ and $T$ are the reflectivity and 

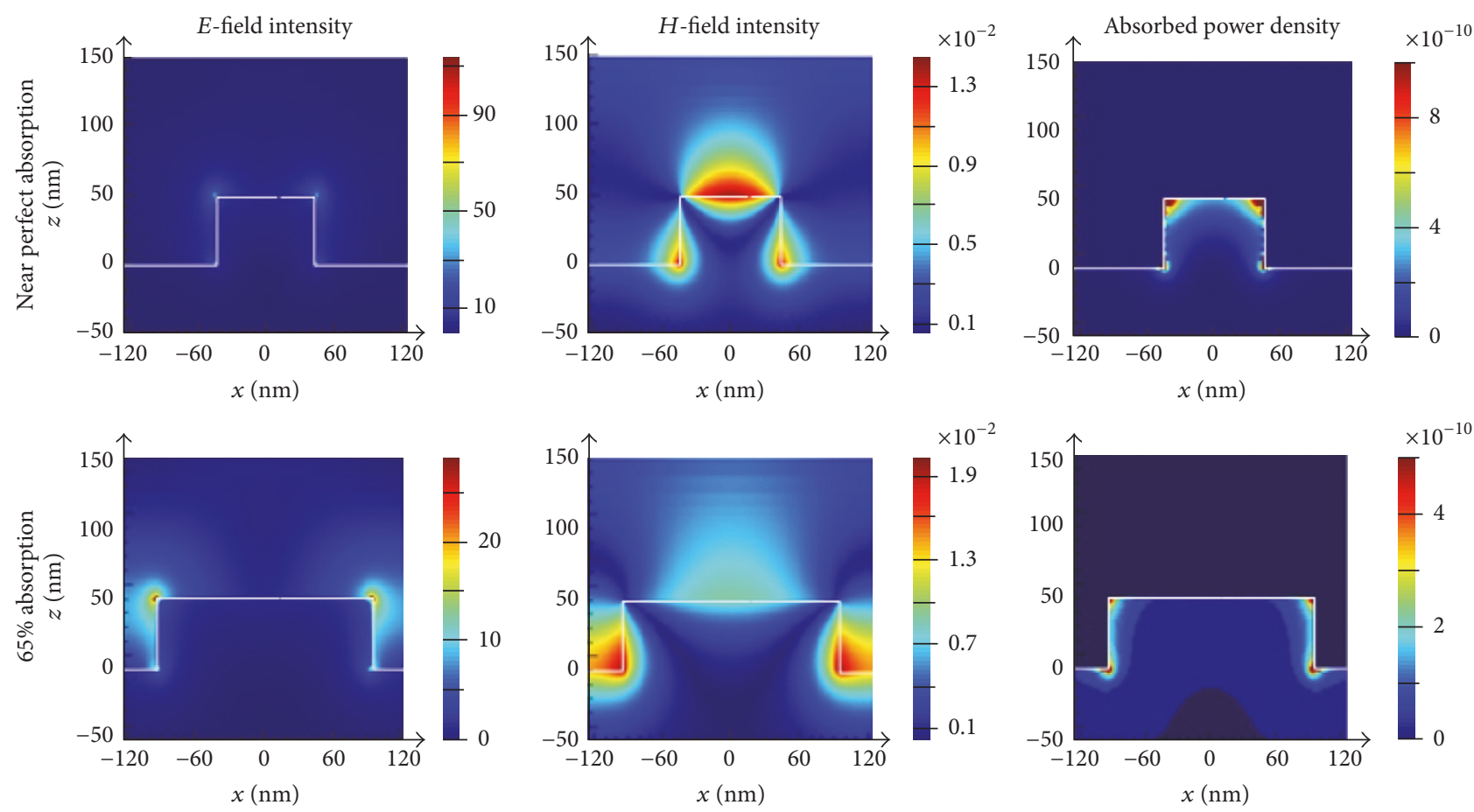

FiguRE 20: Electric and magnetic field and absorbed power density for a structure with nearly perfect maximum absorption at the resonance frequency and a structure with $65 \%$ maximum absorption [70].

transmission, respectively. Since $T$ is 0 due to the optically thick Ag film, naturally, for a perfect reflector we require that the absorption is 0 . In order to achieve a broadband design, the simple linearly changing width of the antenna causes the relative phase shift along the metasurfaces to be continuously modulated. Because the thickness of the antenna is of deep-subwavelength scale the light propagation path can be neglected and the phase discontinuity is only induced by the ultrathin metasurface. This produces a reflection of the beam to an arbitrary direction determined by the gradient of the phase change, and since in the configuration described in [82] there is no spatial-variant phase shift in the $x$-direction, generalised Snell's law for reflection [83] is reduced to

$$
\theta_{r}=\arcsin \left(\frac{\lambda_{0}}{2 \pi} \frac{d \phi}{d y}\right)
$$

where $\theta_{r}$ is the angle of reflection, $\lambda_{0}$ is the vacuum wavelength, and $d \phi / d y$ is the phase shift along the $y$-axis of the interface. This produces an additional effective wave vector to the reflected photons; thus different wavelengths of light are reflected at different angles, giving a rainbow anomalous reflection $[87,88]$.

This design exhibits high broadband reflectivity with an average of over $86 \%$ between 450 and $800 \mathrm{~nm}$. This type of reflector device could be providing functionality in such practical settings as a polarisation beam splitter and high efficiency plasmon coupler as well as flat lenses [80, 89-91] and mirrors [92].
3.2. All Dielectric Silicon Cylinder. Due to the aforementioned shortcomings of plasmonic metamaterials, all dielectric metamaterials utilising Mie resonances [48] in high permittivity dielectric resonators offer a great choice as a low loss alternative at optical frequencies. Additionally, metallic based nanosurfaces are often based on complicated and difficult to produce patterns that require the use of techniques such as electron beam lithography to be fabricated $[49,50,93]$. These techniques are not only slow but expensive which prohibits them from being used on a larger scale. Usually dielectric based metamaterials are based on simpler unit cell geometries such as spheres, cubes, and rods, so they can be patterned with alternative and cheaper techniques, allowing them to be scaled to large areas [94-98].

Recent work by Moitra et al. [98] designed an all dielectric perfect reflector at telecommunications frequencies, based on silicon cylinder resonators and fabricated using self-assembly based nanosphere lithography [99, 100]. While there is, certainly, disorder originating from the self-assembly process, an average reflectance of $99.7 \%$ at $1530 \mathrm{~nm}$ was measured. This is actually higher than the reflectance of metallic mirrors.

\section{Conclusion}

Here we presented a variety of perfect light absorbing and reflecting mechanisms and device designs with a range of functionalities and working frequencies, from $\mathrm{THz}$ to visible light. The key to unlocking effective and efficient perfect absorbers and reflectors is found in the electric and 


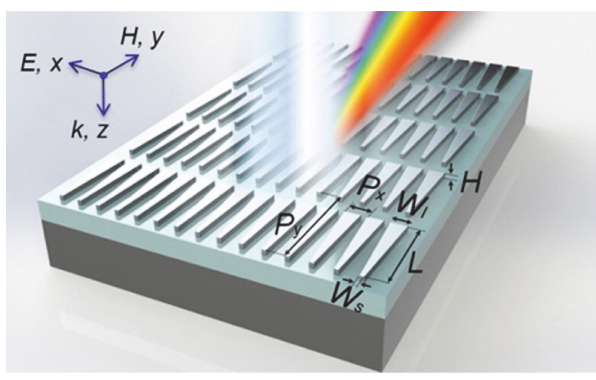

(a)

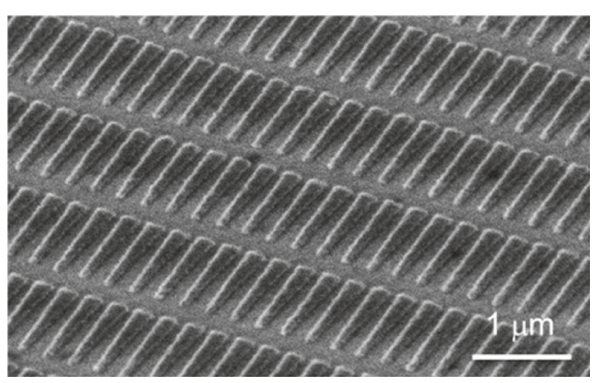

(b)

Figure 21: (a) Schematic drawing of trapezoid shaped nanorod array. (b) SEM image of the Au trapezoid nanorods on top of $\mathrm{SiO}_{2}$ and $\mathrm{Ag}$ substrate [82].

magnetic resonances of the metamaterial or metasurface. By carefully designing the metasurface we can satisfy the impedance matching condition, $\mu(\omega)=\varepsilon(\omega)$, of the magnetic permeability and electric permittivity. By finely tuning the dimensions and design of the metasurface we have the ability to precisely control these parameters.

By changing the structuring of the surface, we have the capability of switching between polarisation dependent and independence. In a device discussed here, it has been shown that this freedom of polarisation could be of great use for applications such as sensing. Incident angle independence has also been shown in many different applications which is a valuable characteristic for devices such as solar cells, but not always a necessary feature. By combining gratings within such devices, it has been shown that we can also recover incident angle dependence at will.

Both broadband and narrowband perfect absorbers have numerous useful applications. One of the main focuses is to address the limitations of metallic absorbers by incorporating dielectric material into the designs, thereby reducing losses due to ohmic losses, heat generation in the metal, and the excitation of surface plasmons at higher frequencies. Ultrabroadband metasurfaces could open the possibilities of creating optically thin coatings that can be designed to have spectral properties as we desire over large spectral ranges, leading to applications such as camouflage, control of radiative heat transfer, and structural colouration. At the other extreme, very narrowband absorbers have applications as incredibly sensitive selective thermal emitters or sensors. If we can engineer the metasurface to have a very sharp and narrowband response, a small change in the surrounding airs makeup could be detected via the impedance matching condition being broken.

Perfect reflectors made of purely dielectric materials have been realised but, as with most of the perfect absorbers discussed here, are still expensive, difficult, and time consuming to fabricate. A future focus for perfect reflectors is a hybrid, metallic. and dielectric, perfect reflector where the dielectric layer would act as a support to the already perfect metal reflector; this would lead to an increase of their versatility and longevity in practical use compared to just metallic mirrors.

In summary, scalable, highly tuneable, active, and easy to fabricate metasurfaces are the holy grail for all the field. By utilising dielectric materials in combination with metals, we are heading towards highly tuneable and robust metasurfaces but at the moment we are hindered by the limitations of the current, slow fabrication processes. There are further potential directions to be explored that were not covered in this paper, such as the use of liquid crystals in strategic locations in the metamaterial and the use of phase change materials. Both of these approaches add an extra degree of freedom to the tunability of the metasurface that can be controlled electrically or optically. The advancement of perfect absorbers and reflectors has a great deal of real world applicability and could pave the way for future technological advances in many fields.

\section{Conflicts of Interest}

The authors declare that they have no conflicts of interest.

\section{Acknowledgments}

This work is financially supported by the National Research Foundation of Korea (NRF) grant funded by the Ministry of Science, ICT and Future Planning (MSIP) of Korean government (CAMM-2014M3A6B3063708 and NRF2015R1A5A1037668).

\section{References}

[1] Y. Avitzour, Y. Urzhumov, and G. Shvets, "Wide-angle infrared absorber based on a negative-index plasmonic metamaterial," Physical Review B, vol. 79, Article ID 045131, 2009.

[2] X. Liu, T. Tyler, T. Starr, A. F. Starr, N. M. Jokerst, and W. J. Padilla, "Taming the blackbody with infrared metamaterials as selective thermal emitters," Physical Review Letters, vol. 107, no. 4, Article ID 045901, 2011.

[3] T. V. Teperik, F. J. García De Abajo, A. G. Borisov et al., "Omnidirectional absorption in nanostructured metal surfaces," Nature Photonics, vol. 2, no. 5, pp. 299-301, 2008.

[4] B. J. Lee and Z. M. Zhang, "Design and fabrication of planar multilayer structures with coherent thermal emission characteristics," Journal of Applied Physics, vol. 100, no. 6, Article ID 063529, 2006.

[5] K. Aydin, V. E. Ferry, R. M. Briggs, and H. A. Atwater, "Broadband polarization-independent resonant light absorption using 
ultrathin plasmonic super absorbers," Nature Communications, vol. 2, no. 1, Article ID 517, 2011.

[6] Y. Q. Ye, Y. Jin, and S. He, "Omnidirectional, polarizationinsensitive and broadband thin absorber in the terahertz regime," Journal of the Optical Society of America B, vol. 27, no. 3, pp. 498-504, 2010.

[7] W. Wang, Y. Cui, Y. He et al., "Efficient multiband absorber based on one-dimensional periodic metal-dielectric photonic crystal with a reflective substrate," Optics Letters, vol. 39, no. 2, pp. 331-334, 2014.

[8] J. Yu, Y. Shen, X. Liu, R. Fu, J. Zi, and Z. Zhu, "Absorption in one-dimensional metallic-dielectric photonic crystals," Journal of Physics: Condensed Matter, vol. 16, no. 7, Article ID L51, 2004.

[9] N. Mattiucci, M. J. Bloemer, N. Akozbek, and G. D’Aguanno, "Impedance matched thin metamaterials make metals absorbing," Scientific Reports, vol. 3, Article ID 3203, 2013.

[10] D. Schurig, J. J. Mock, B. J. Justice et al., "Metamaterial electromagnetic cloak at microwave frequencies," Science, vol. 314, no. 5801, pp. 977-980, 2006.

[11] D. Maystre and R. Petit, "Brewster incidence for metallic gratings," Optics Communications, vol. 17, no. 2, pp. 196-200, 1976.

[12] M. C. Hutley and D. Maystre, "The total absorption of light by a diffraction grating," Optics Communications, vol. 19, no. 3, pp. 431-436, 1976.

[13] M. Nevière, D. Maystre, R. MacPhedran, G. Derrick, and M. Hutley, in Proceeding of the 11th Conference of the International Commission for Optics ICO-11, Madrid, Spain, September 1978.

[14] G. H. Derrick, R. C. McPhedran, D. Maystre, and M. Nevière, "Crossed gratings: a theory and its applications," Journal of Applied Physics, vol. 18, no. 1, pp. 39-52, 1979.

[15] J.-J. Greffet, R. Carminati, K. Joulain, J.-P. Mulet, S. Mainguy, and Y. Chen, "Coherent emission of light by thermal sources," Nature, vol. 416, no. 6876, pp. 61-64, 2002.

[16] S. Collin, F. Pardo, R. Teissier, and J. L. Pelouard, "Efficient light absorption in metal-semiconductormetal nanostructures," Applied Physics Letters, vol. 85, pp. 194-196, 2004.

[17] Y. P. Bliokh, J. Felsteiner, and Y. Z. Slutsker, "Total absorption of an electromagnetic wave by an overdense plasma," Physical Review Letters, vol. 95, no. 16, Article ID 165003, 2005.

[18] M. E. Abdelsalam, P. N. Bartlett, J. J. Baumberg, and S. Coyle, "Preparation of arrays of isolated spherical cavities by self-assembly of polystyrene spheres on self-assembled prepatterned macroporous films," Advanced Materials, vol. 16, no. 1, pp. 90-93, 2004.

[19] N. I. Landy, S. Sajuyigbe, J. J. Mock, D. R. Smith, and W. J. Padilla, "Perfect metamaterial absorber," Physical Review Letters, vol. 100, no. 20, Article ID 207402, 2008.

[20] H. Tao, N. I. Landy, C. M. Bingham, X. Zhang, R. D. Averitt, and W. J. Padilla, "A metamaterial absorber for the terahertz regime: design, fabrication and characterization," Optics Express, vol. 16, no. 10, pp. 7181-7188, 2008.

[21] Y. Q. Ye, Y. Jin, and S. He, "Omnidirectional, polarizationinsensitive and broadband thin absorber in the terahertz regime," Journal of the Optical Society of America B: Optical Physics, vol. 27, no. 3, pp. 498-504, 2010.

[22] N. Engheta, "Thin absorbing screens using metamaterial surfaces," in Proceedings of the IEEE Antennas and Propagation Society International Symposium, vol. 2, pp. 392-395, San Antonio, Tex, USA, June 2002.
[23] H. Tao, C. M. Bingham, A. C. Strikwerda et al., "Highly flexible wide angle of incidence terahertz metamaterial absorber: design, fabrication, and characterization," Physical Review B: Condensed Matter and Materials Physics, vol. 78, no. 24, Article ID 241103, 2008.

[24] N. Liu, L. Fu, S. Kaiser, H. Schweizer, and H. Giessen, "Plasmonic building blocks for magnetic molecules in threedimensional optical metamaterials," Advanced Materials, vol. 20, no. 20, pp. 3859-3865, 2008.

[25] T. Li, H. Liu, F. M. Wang, Z. G. Dong, S. N. Zhu, and X. Zhang, "Coupling effect of magnetic polariton in perforated metal/dielectric layered metamaterials and its influence on negative refraction transmission," Optics Express, vol. 14, no. 23, pp. 11155-11163, 2006.

[26] N. Liu, H. Guo, L. Fu, S. Kaiser, H. Schweizer, and H. Giessen, "Plasmon hybridization in stacked cut-wire metamaterials," Advanced Materials, vol. 19, no. 21, pp. 3628-3632, 2007.

[27] J. Zhou, L. Zhang, G. Tuttle, T. Koschny, and C. M. Soukoulis, "Negative index materials using simple short wire pairs," Physical Review B: Condensed Matter and Materials Physics, vol. 73, no. 4, Article ID 041101, 2006.

[28] A. Boltasseva and V. M. Shalaev, "Fabrication of optical negative-index metamaterials: recent advances and outlook," Metamaterials, vol. 2, no. 1, pp. 1-17, 2008.

[29] M. Qi, E. Lidorikis, P. T. Rakich et al., "A three-dimensional optical photonic crystal with designed point defects," Nature, vol. 429, no. 6991, pp. 538-542, 2004.

[30] G. Subramania and S. Y. Lin, "Fabrication of three-dimensional photonic crystal with alignment based on electron beam lithography," Applied Physics Letters, vol. 85, no. 21, pp. 5037-5039, 2004.

[31] A. S. P. Chang, Y. S. Kim, M. Chen et al., "Visible threedimensional metallic photonic crystal with non-localized propagating modes beyond waveguide cutoff,' Optics Express, vol. 15, no. 13, pp. 8428-8437, 2007.

[32] N. Liu, M. Mesch, T. Weiss, M. Hentschel, and H. Giessen, "Infrared perfect absorber and its application as plasmonic sensor," Nano Letters, vol. 10, no. 7, pp. 2342-2348, 2010.

[33] X. L. Liu, T. Starr, A. F. Starr, and W. J. Padilla, "Infrared spatial and frequency selective metamaterial with near-unity absorbance," Physical Review Letters, vol. 104, Article ID 207403, 2010.

[34] V. A. Fedotov, P. L. Mladyonov, S. L. Prosvirnin, and N. I. Zheludev, "Planar electromagnetic metamaterial with a fish scale structure," Physical Review E: Covering Statistical, Nonlinear, Biological, and Soft Matter Physics, vol. 72, no. 5, Article ID 056613, 2005.

[35] V. A. Fedotov, V. V. Khardikov, S. L. Prosvirnin, Y. Chen, and N. I. Zheludev, "Optical Magnetic Mirrors," Journal of Optics A: Pure and Applied Optics, vol. 9, Article ID L1, 2007.

[36] E. M. Larsson, J. Alegret, M. Kall, and D. S. Sutherland, “Sensing characteristics of NIR localized surface plasmon resonances in gold nanorings for application as ultrasensitive biosensors," Nano Letters, vol. 7, no. 5, pp. 1256-1263, 2007.

[37] N. Feth, C. Enkrich, M. Wegener, and S. Linden, "Large-area magnetic metamaterials via compact interference lithography," Optics Express, vol. 15, no. 2, pp. 501-507, 2007.

[38] M. C. Gwinner, E. Koroknay, F. Liwei et al., "Periodic large-area metallic split-ring resonator metamaterial fabrication based on shadow nanosphere lithography," Small, vol. 5, no. 3, pp. 400406, 2009. 
[39] N. Liu, T. Weiss, M. Mesch et al., "Planar metamaterial analogue of electromagnetically induced transparency for plasmonic sensing," Nano Letters, vol. 10, no. 4, pp. 1103-1107, 2010.

[40] A. Polyakov, S. Cabrini, S. Dhuey, B. Harteneck, P. J. Schuck, and H. A. Padmore, "Plasmonic light trapping in nanostructured metal surfaces," Applied Physics Letters, vol. 98, no. 20, Article ID 203104, 2011.

[41] V. G. Kravets, F. Schedin, and A. N. Grigorenko, "Plasmonic blackbody: Almost complete absorption of light in nanostructured metallic coatings," Physical Review B: Condensed Matter and Materials Physics, vol. 78, no. 20, Article ID 205405, 2008.

[42] L. Meng, D. Zhao, Q. Li, and M. Qiu, "Polarization-sensitive perfect absorbers at near-infrared wavelengths: erratum," Optics Express, vol. 21, pp. A229-A230, 2013.

[43] X. Shen, T. J. Cui, J. Zhao, H. F. Ma, W. X. Jiang, and H. Li, "Polarization-independent wide-angle triple-band metamaterial absorber," Optics Express, vol. 19, no. 10, pp. 9401-9407, 2011.

[44] H. Li, L. H. Yuan, B. Zhou, X. P. Shen, Q. Cheng, and T. J. Cui, "Ultrathin multiband gigahertz metamaterial absorbers," Journal of Applied Physics, vol. 110, no. 1, Article ID 014909, 2011.

[45] H. Tao, C. M. Bingham, D. Pilon et al., "A dual band terahertz metamaterial absorber," Journal of Physics D: Applied Physics, vol. 43, no. 22, Article ID 225102, 2010.

[46] M. H. Li, H. L. Yang, X. W. Hou, Y. Tian, and D.-Y. Hou, "Perfect metamaterial absorber with dual bands," Progress In Electromagnetics Research, vol. 108, pp. 37-49, 2010.

[47] S. Gu, J. P. Barrett, T. H. Hand, B. Popa, and S. A. Cummer, "A broadband low-reflection metamaterial absorber," Journal of Applied Physics, vol. 108, no. 6, Article ID 064913, 2010.

[48] C. M. Soukoulis and M. Wegener, "Past achievements and future challenges in the development of three-dimensional photonic metamaterials," Nature Photonics, vol. 5, no. 9, pp. 523-530, 2011.

[49] A. Boltasseva and H. A. Atwater, "Low-loss plasmonic metamaterials," Science, vol. 331, no. 6015, pp. 290-291, 2011.

[50] N. I. Zheludev, “The road ahead for metamaterials," Science, vol. 328, no. 5978, pp. 582-583, 2010.

[51] H. Deng, Z. Li, L. Stan et al., "Broadband perfect absorber based on one ultrathin layer of refractory metal," Optics Expresss, vol. 40, no. 11, pp. 2592-2595, 2015.

[52] H. A. Haus, Waves and Fields in Optoelectronics, Prentice Hall, Upper Saddle River, NJ, USA, 1984.

[53] E. D. Palik, Handbook of Optical Constants of Solids, Academic Press, Cambridge, Mass, USA, 1998.

[54] D. Barchiesi and T. Grosges, "Fitting the optical constants of gold, silver, chromium, titanium, and aluminum in the visible bandwidth," Journal of Nanophotonics, vol. 8, no. 1, Article ID 083097, 2014.

[55] A. D. Rakić, A. B. Djurišić, J. M. Elazar, and M. L. Majewski, "Optical properties of metallic films for vertical-cavity optoelectronic devices," Applied Optics, vol. 37, no. 22, pp. 5271-5283, 1998.

[56] P. Buffat and J.-P. Borel, "Size effect on the melting temperature of gold particles," Physical Review A: Covering Atomic, Molecular, and Optical Physics and Quantum Information, vol. 13, no. 6, pp. 2287-2298, 1976.

[57] N. I. Landy, C. M. Bingham, T. Tyler, N. Jokerst, D. R. Smith, and W. J. Padilla, "Design, theory, and measurement of a polarization-insensitive absorber for terahertz imaging," Physical Review B: Condensed Matter and Materials Physics, vol. 79, no. 12, Article ID 125104, 2009.
[58] M. Laroche, G. Arnold, F. Marquier et al., "Highly directional radiation generated by a tungsten thermal source," Optics Expresss, vol. 30, no. 19, pp. 2623-2625, 2005.

[59] R. W. Wood, "On a remarkable case of uneven distribution of light in a diffraction grating spectrum," Philosophical Magazine, vol. 4, Article ID 396, 1902.

[60] A. Sharon, S. Glasberg, D. Rosenblatt, and A. A. Friesem, "Metal-based resonant grating waveguide structures," Journal of the Optical Society of America A, vol. 14, no. 3, pp. 588-595, 1997.

[61] L. Meng, D. Zhao, Z. Ruan, Q. Li, Y. Yang, and M. Qiu, "Optimized grating as an ultra-narrow band absorber or plasmonic sensor,” Optics Expresss, vol. 39, no. 5, pp. 1137-1140, 2014.

[62] Z. Ruan and S. Fan, "Temporal coupled-mode theory for fano resonance in light scattering by a single obstacle," The Journal of Physical Chemistry C, vol. 114, no. 16, pp. 7324-7329, 2010.

[63] J. Yoon, K. H. Seol, S. H. Song, and R. Magnusson, "Critical coupling in dissipative surface-plasmon resonators with multiple ports," Optics Express, vol. 18, no. 25, pp. 25702-25711, 2010.

[64] J. Wu, C. Zhou, J. Yu, H. Cao, S. Li, and W. Jia, “TE polarization selective absorber based on metal-dielectric grating structure for infrared frequencies," Optics Communications, vol. 329, pp. 38-43, 2014.

[65] J. Wu, C. H. Zhou, H. C. Cao, and A. D. Hu, "Polarizationdependent and -independent spectrum selective absorption based on a metallic grating structure," Optics Communications, vol. 309, pp. 57-71, 2013.

[66] M. A. Kats, R. Blanchard, P. Genevet, and F. Capasso, "Nanometre optical coatings based on strong interference effects in highly absorbing media," Nature Materials, vol. 12, no. 1, pp. 20-24, 2013.

[67] C.-H. Lin, R.-L. Chern, and H.-Y. Lin, "Polarizationindependent broad-band nearly perfect absorbers in the visible regime," Optics Express, vol. 19, no. 2, pp. 415-424, 2011.

[68] W. Li and J. Valentine, "Metamaterial perfect absorber based hot electron photodetection," Nano Letters, vol. 14, no. 6, pp. 35103514, 2014.

[69] F. Cheng, J. Gao, S. T. Luk, and X. Yang, "Structural color printing based on plasmonic metasurfaces of perfect light absorption," Scientific Reports, vol. 5, Article ID 11045, 2015.

[70] R. J. H. Ng, X. M. Goh, and J. K. W. Yang, "All-metal nanostructured substrates as subtractive color reflectors with near-perfect absorptance," Optics Express, vol. 23, no. 25, pp. 32597-32605, 2015.

[71] J. Zhang, J.-Y. Ou, N. Papasimakis, Y. Chen, K. F. MacDonald, and N. I. Zheludev, "Continuous metal plasmonic frequency selective surfaces," Optics Express, vol. 19, no. 23, pp. 2327923285, 2011.

[72] T. Søndergaard, S. M. Novikov, T. Holmgaard et al., "Plasmonic black gold by adiabatic nanofocusing and absorption of light in ultra-sharp convex grooves," Nature Communications, vol. 3, Article ID 969, 2012.

[73] L. T. Varghese, L. Fan, Y. Xuan, C. Tansarawiput, S. Kim, and M. Qi, "Resistless nanoimprinting in metal for plasmonic nanostructures," Small, vol. 9, no. 22, pp. 3778-3783, 2013.

[74] J. S. Clausen, E. Højlund-Nielsen, A. B. Christiansen et al., "Plasmonic metasurfaces for coloration of plastic consumer products," Nano Letters, vol. 14, no. 8, pp. 4499-4504, 2014.

[75] X. M. Goh, Y. Zheng, S. J. Tan et al., “Three-dimensional plasmonic stereoscopic prints in full colour," Nature Communications, vol. 5, Article ID 5361, 2015. 
[76] S. J. Tan, L. Zhang, D. Zhu et al., "Plasmonic color palettes for photorealistic printing with aluminum nanostructures," Nano Letters, vol. 14, no. 7, pp. 4023-4029, 2014.

[77] A. B. Christiansen, G. P. Caringal, J. S. Clausen et al., "Black metal thin films by deposition on dielectric antireflective motheye nanostructures," Scientific Reports, vol. 5, Article ID 10563, 2015.

[78] B. Slovick, Z. G. Yu, M. Berding, and S. Krishnamurthy, "Perfect dielectric-metamaterial reflector," Physical Review B, vol. 88, no. 16, Article ID 165116, 2013.

[79] S. Sun, K.-Y. Yang, C.-M. Wang et al., "High-efficiency broadband anomalous reflection by gradient meta-surfaces," Nano Letters, vol. 12, no. 12, pp. 6223-6229, 2012.

[80] A. Pors, M. G. Nielsen, R. L. Eriksen, and S. I. Bozhevolnyi, "Broadband focusing flat mirrors based on plasmonic gradient metasurfaces," Nano Letters, vol. 13, no. 2, pp. 829-834, 2013.

[81] S. Sun, Q. He, S. Xiao, Q. Xu, X. Li, and L. Zhou, "Gradientindex meta-surfaces as a bridge linking propagating waves and surface waves," Nature Materials, vol. 11, pp. 426-431, 2012.

[82] Z. Li, E. Palacios, S. Butun, and K. Aydin, "Visible-frequency metasurfaces for broadband anomalous reflection and highefficiency spectrum splitting," Nano Letters, vol. 15, no. 3, pp. 1615-1621, 2015.

[83] N. Yu, P. Genevet, M. A. Kats et al., "Light propagation with phase discontinuities: Generalized laws of reflection and refraction," Science, vol. 334, no. 6054, pp. 333-337, 2011.

[84] N. Yu and F. Capasso, "Flat optics with designer metasurfaces," Nature Materials, vol. 13, no. 2, pp. 139-150, 2014.

[85] S. Butun and K. Aydin, "Structurally tunable resonant absorption bands in ultrathin broadband plasmonic absorbers," Optics Express, vol. 22, no. 16, pp. 19457-19468, 2014.

[86] Z. Li, S. Butun, and K. Aydin, “Touching Gold Nanoparticle Chain Based Plasmonic Antenna Arrays and Optical Metamaterials," ACS Photonics, vol. 1, no. 3, pp. 228-234, 2014.

[87] L. Zhang, J. Hao, M. Qiu, S. Zouhdi, J. K. W. Yang, and C.W. Qiu, "Anomalous behavior of nearly-entire visible band manipulated with degenerated image dipole array," Nanoscale, vol. 6, no. 21, pp. 12303-12309, 2014.

[88] Z. Li, L. Huang, K. Lu, Y. Sun, and L. Min, "Continuous metasurface for high-performance anomalous reflection," Applied Physics Express, vol. 7, Article ID 112001, 2014.

[89] D. Lin, P. Fan, E. Hasman, and M. L. Brongersma, "Dielectric gradient metasurface optical elements," Science, vol. 345, no. 6194, pp. 298-302, 2014.

[90] X. Chen, L. Huang, H. Mühlenbernd et al., "Dual-polarity plasmonic metalens for visible light," Nature Communications, vol. 3, Article ID 1198, 2012.

[91] E. T. F. Rogers, J. Lindberg, T. Roy et al., "A super-oscillatory lens optical microscope for subwavelength imaging," Nature Materials, vol. 11, no. 5, pp. 432-435, 2012.

[92] P. Moitra, B. A. Slovick, Z. Gang Yu, S. Krishnamurthy, and J. Valentine, "Experimental demonstration of a broadband all-dielectric metamaterial perfect reflector," Applied Physics Letters, vol. 104, no. 17, Article ID 171102, 2014.

[93] P. Moitra, Y. Yang, Z. Anderson, I. I. Kravchenko, D. P. Briggs, and J. Valentine, "Realization of an all-dielectric zero-index optical metamaterial," Nature Photonics, vol. 7, no. 10, pp. 791795, 2013.

[94] K. Vynck, D. Felbacq, E. Centeno, A. I. Cǎbuz, D. Cassagne, and B. Guizal, "All-dielectric rod-type metamaterials at optical frequencies," Physical Review Letters, vol. 102, no. 13, Article ID 133901, 2009.
[95] B. I. Popa and S. A. Cummer, "Compact dielectric particles as a building block for low-loss magnetic metamaterials," Physical Review Letters, vol. 100, no. 20, Article ID 207401, 2008.

[96] Y. H. Fu, A. I. Kuznetsov, A. E. Miroshnichenko, Y. F. Yu, and B. Luk'yanchuk, "Directional visible light scattering by silicon nanoparticles," Nature Communications, vol. 4, Article ID 1527, 2013.

[97] A. García-Etxarri, R. Gómez-Medina, L. S. Froufe-Pérez et al., "Strong magnetic response of submicron Silicon particles in the infrared," Optics Express, vol. 19, no. 6, pp. 4815-4826, 2011.

[98] P. Moitra, B. A. Slovick, W. Li et al., "Large-Scale All-Dielectric Metamaterial Perfect Reflectors," ACS Photonics, vol. 2, no. 6, pp. 692-698, 2015.

[99] C. F. Bohren and D. R. Huffman, Absorption and Scattering of Light by Small Particles, John Wiley \& Sons, Hoboken, NJ, USA, 1983.

[100] D. M. Nguyen, D. Lee, and J. Rho, "Control of light absorbance using plasmonic grating based perfect absorber at visible and near-infrared wavelengths," Scientific Reports, vol. 7, no. 1, 2017. 

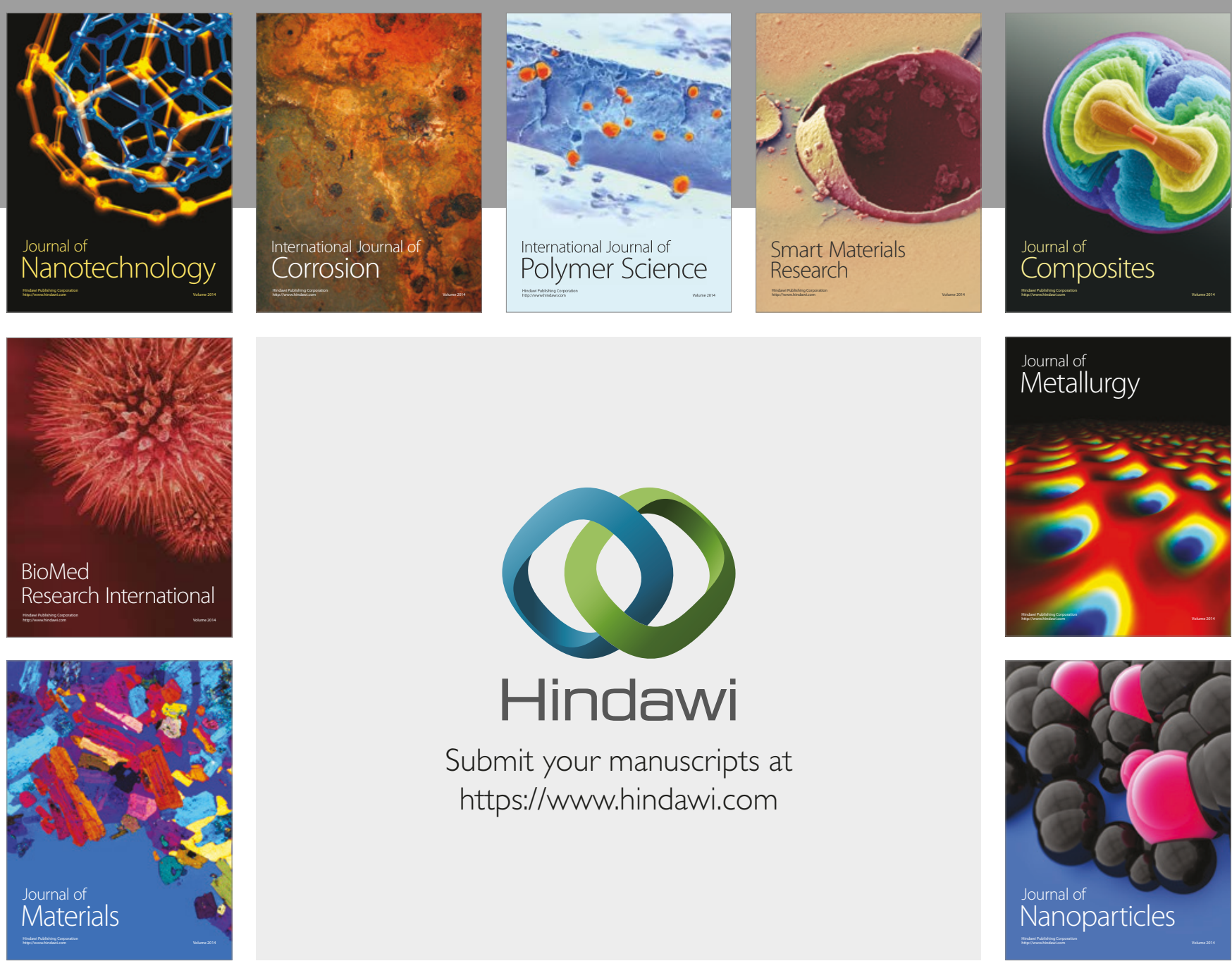

\section{Hindawi}

Submit your manuscripts at

https://www.hindawi.com
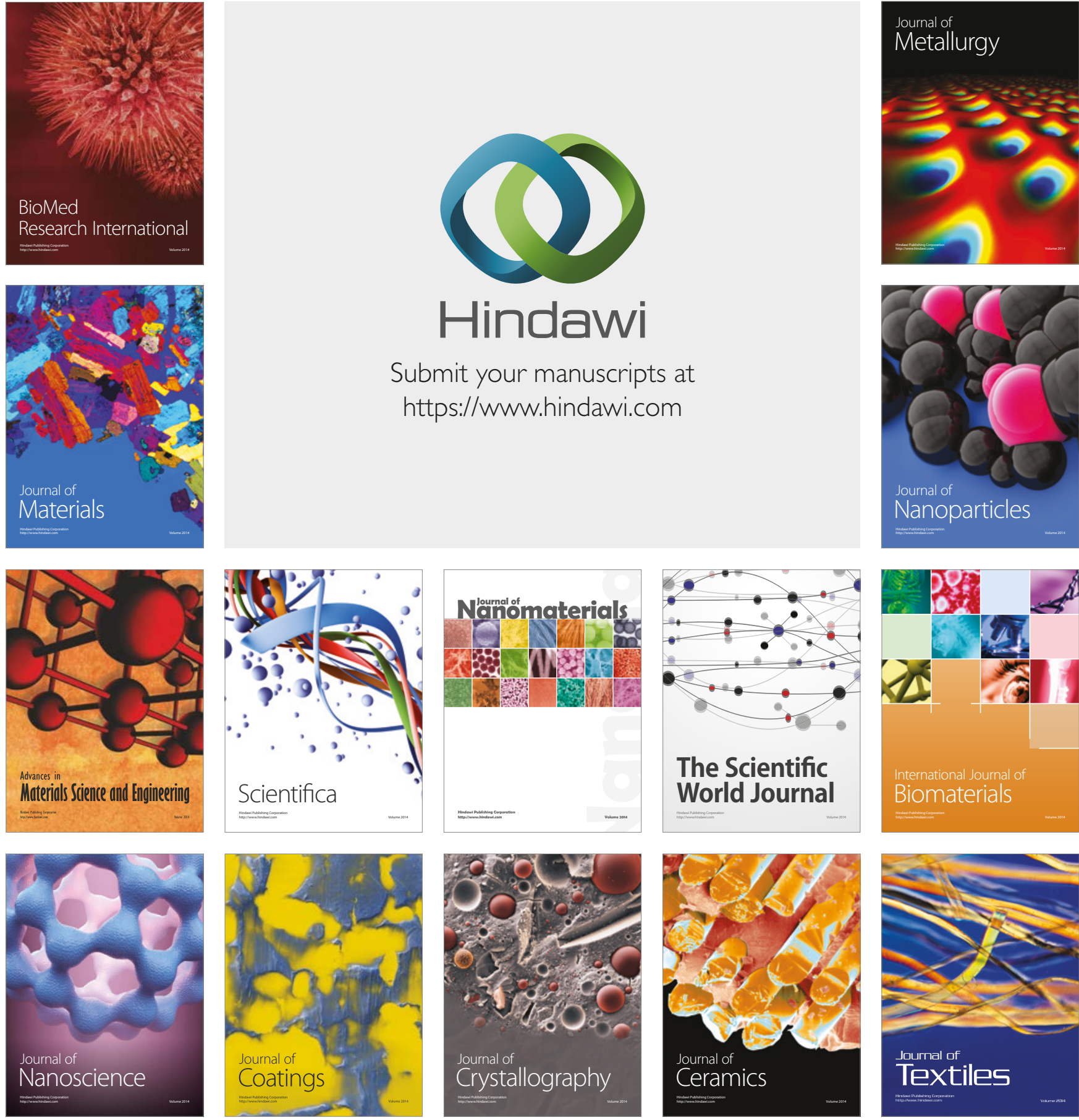

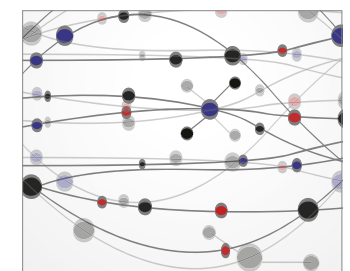

The Scientific World Journal
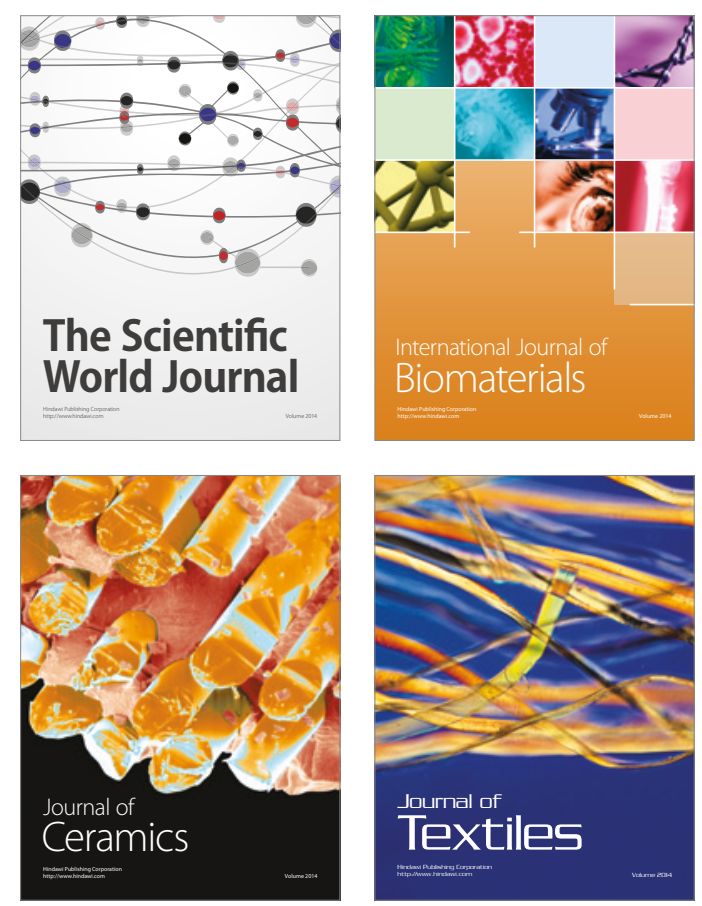